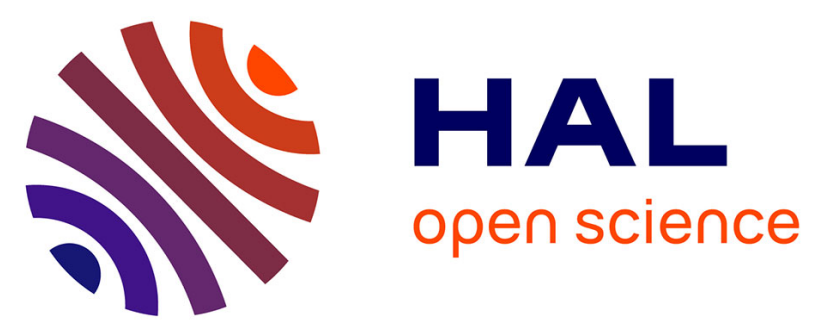

\title{
Safe navigation in a coastal environment of multiple surface vehicles under uncertainties: A combined use of potential field constructions and NMPC
}

Ngo-Quoc-Huy Tran, Ionela Prodan, Esten Ingar Grøtli, Laurent Lefèvre

\section{- To cite this version:}

Ngo-Quoc-Huy Tran, Ionela Prodan, Esten Ingar Grøtli, Laurent Lefèvre. Safe navigation in a coastal environment of multiple surface vehicles under uncertainties: A combined use of potential field constructions and NMPC. Ocean Engineering, 2020, 216, pp.107706. 10.1016/j.oceaneng.2020.107706 . hal-03100171

\section{HAL Id: hal-03100171 \\ https://hal.science/hal-03100171}

Submitted on 6 Jan 2021

HAL is a multi-disciplinary open access archive for the deposit and dissemination of scientific research documents, whether they are published or not. The documents may come from teaching and research institutions in France or abroad, or from public or private research centers.
L'archive ouverte pluridisciplinaire HAL, est destinée au dépôt et à la diffusion de documents scientifiques de niveau recherche, publiés ou non, émanant des établissements d'enseignement et de recherche français ou étrangers, des laboratoires publics ou privés. 


\title{
Safe navigation in a coastal environment of multiple surface vehicles under uncertainties: a combined use of potential field constructions and NMPC
}

\author{
Ngo-Quoc-Huy Tran, Ionela Prodana , Esten Ingar Grøtlii , Laurent Lefèvre ${ }^{a}$ \\ ${ }^{a}$ Univ. Grenoble Alpes, Grenoble INP, LCIS, F-26000, Valence \\ ${ }^{b}$ Mathematics and Cybernetics, SINTEF Digital, Norway
}

\begin{abstract}
This paper deals with the motion planning problem for surface vehicles in the presence of varying environmental disturbances. The goal is to steer the vehicles from an initial to a final destination while ensuring connectivity and avoiding collision with fixed and moving obstacles. The novelty lies in the coherent combination among various ingredients for path generation and potential field constructions for collision avoidance and connectivity maintenance, all gathered in a distributed NMPC (Nonlinear Model Predictive Control) framework: i) LOS (Line-of-Sight) guidance and RRT* (optimal Rapidly-exploring Random Tree) algorithms are employed for generating a collision-free path considering static surroundings; ii) on-off barrier functions activate the proposed potential field components which are in the view range of the agents, hence ensuring safe navigation in a dynamic coastal environment with a low computation demand; iii) a NDO (Nonlinear Disturbance Observer) is integrated in the NMPC design to compensate the environmental disturbances. The proposed algorithm is validated through simulations and comparisons carried out over a benchmark for USVs (Unmanned Surface Vehicles) safe navigation in the Trondheim fjord, Norway.
\end{abstract}

Keywords: Surface vehicles, Connectivity maintenance and collision

Email addresses: ngo-quoc-huy.tran@lcis.grenoble-inp.fr (Ngo-Quoc-Huy Tran), ionela.prodan@lcis.grenoble-inp.fr (Ionela Prodan), EstenIngar.Grotli@sintef .no (Esten Ingar Grøtli), laurent. lefevre@lcis .grenoble-inp.fr (Laurent Lefèvre) 
avoidance, Potential field constructions, Nonlinear disturbance observer, NMPC.

\section{Notations}

The following notation will be used throughout the paper. For a vector $x \in \mathbb{R}^{n}$ and a positive definite matrix $P \in \mathbb{R}^{n \times n},\|x\|_{P}$ denotes the weighted norm $\sqrt{x^{T} P x}, \| x||$ the Euclidean norm and $|x|$ the absolute value of the vector ${ }_{5} x$. A polytope is a bounded polyhedron and has a dual representation in terms of intersection of half-spaces or convex hull of extreme points: $\mathcal{P}=\left\{x \in \mathbb{R}^{n}\right.$ : $S x \leq K\}=\left\{x \in \mathbb{R}^{n}: x=\sum \alpha_{i} v_{i}, \sum \alpha_{i}=1, \alpha_{i} \geq 0\right\}$.

Table 1: List of variables.

\begin{tabular}{ll}
\hline Notation & Description \\
\hline $\mathcal{T}_{u_{i}}$ & surge thrust \\
$\mathcal{T}_{r_{i}}$ & rudder deflection \\
$u_{i}$ & surge velocity of the $i^{\text {th }}$ ship \\
$v_{i}$ & sway velocity of the $i^{\text {th }}$ ship \\
$r_{i}$ & yaw rate of the $i^{\text {th }}$ ship \\
$\psi_{i}$ & heading or yaw angle of the $i^{\text {th }}$ ship \\
$w_{i}$ & disturbance of the $i^{\text {th }}$ ship model \\
$\rho_{i}$ & view range of the $i^{\text {th }}$ ship \\
$\left\|\Delta p_{i, \ell}^{s}\right\|$ & Euclidean distance calculated from Chebyshev center, the largest \\
& inscribed ball of $\ell^{\text {th }}$ polytope and current position of the $i^{\text {th }}$ agent \\
$\left\|\Delta p_{i, j}^{d}\right\|$ & Euclidean distance between the current position of $i^{t h}$ and $j^{t h}$ agents \\
$\delta_{p}(\cdot)$ & the orientation of the desired path \\
$x_{e}, y_{e}$ & along-track and cross-track error \\
$\alpha$ & parameterization variable for establishing the desired path \\
$\Lambda$ & look-ahead distance \\
$\psi_{i L O S}$ & yaw angle's reference obtained by the LOS guidance system \\
$\hat{v}_{i}$ & estimated acceleration of the $i^{\text {th }}$ ship
\end{tabular}




$\begin{array}{ll}v_{i e} & \text { acceleration error of the } i^{\text {th }} \text { ship } \\ \hat{w}_{i} & \text { estimated disturbance of the } i^{\text {th }} \text { ship } \\ \hat{w}_{i e} & \text { disturbance error of the } i^{t h} \text { ship }\end{array}$

\section{Introduction}

\subsection{Motivation} surface vehicles; guaranteeing connectivity maintenance among the ships for information exchange in the course of performing tasks and rejecting the external disturbance from the ocean which may highly affect the performance of the USVs. ning of multi-surface vehicles complying with the COLREGS rules while ensuring connectivity and collision avoidance (with fixed and mobile obstacles).

\subsection{Related work and remaining open problems}

The classical problem of collision avoidance, which is formulated in terms of non-convex constraints added in the control optimization problem, is usually addressed through: 
i) the use of MIP (Mixed-Integer Programming) where the constraints are explicitly taken into account but with the cost of a high computation time [5, 6]. Some maritime applications using MIP are, for example: determining efficient schedules for USVs/UUVs (Unmanned Underwater Vehicles) in performing a mine countermeasure mission [7], reconfigurable USVs [8];

ii) the use of indirect methods based on potential field constructions which show good computational performances [9, 10] but where the local minima issue is still a shortcoming of these approaches. [11, 12, 13] show the effectiveness in dealing with obstacle and collision avoidance when APF (Artificial Potential Field) is used to establish feasible paths for USVs.

In addition, COLREGS-compliance for safe maritime navigation should also be addressed and various approaches for this problem have been tested in the literature: velocity obstacle [14], rapidly-exploring random tree [15] or model predictive control, binary variables to choose scenarios with multi-moving obstacles with erratic motion [16], using a rolling horizon optimization approach to find optimal heading angles for collision avoidance with other ships [17], slack variables to change the vessel's direction for specific situations [18] or elliptical ship domain [19], distributed coordination based on constraint optimization [20], [21]. These approaches consider disc-like approximation for the static or dynamic obstacles, which might be too conservative, w.r.t the conditions in a real environment.

Another challenging issue in motion planning for multi-agent systems is the connectivity maintenance, which ensures that the information exchange and sharing can be reliably realized by forwarding messages among agents while implementing and allocating tasks. Some applications can be found in a variety of practical tasks, such as search and rescue, surveillance, cooperative transportation by autonomous vehicles at sea or on the ground, attitude alignment of clusters of satellites, air traffic management system, etc. Related to this problem, there are some recent works for always ensuring that the agents lies 
within their communication range. In general, we may classify them in two directions:

1. imposing hard constraints based on the Euclidean distance between agents, which must be less than their sensing radius as in [22]. This condition is handled in a decentralized NMPC framework. However, this approach is too strict and can lead to infeasibility in complex cases;

2. using a potential-field method to ensure that an algebraic connectivity condition holds (i.e., which refers to the fact that the second-smallest eigenvalue of the Laplacian matrix is strictly greater than zero [23], [24]). Here, the problem of flocking of second-order multi-agent systems is addressed using potential hybrid fields which ensure both connectivity preservation and collision avoidance in a distributed control framework.

We underline that it is necessary to propose robust controllers for multiagent systems to deal with uncertainties [25]. Tube-based MPC is considered as one of the most popular approaches for coping with perturbations [26, 27]. The general idea is to maintain the actual state within a safety region along the optimal state trajectory [28]. Another approach is to design a disturbance observer to reject the disturbances leading to an improved system robustness [29, 30]. The authors in [31] propose a nonlinear observer within an NMPC scheme for a SVC (Static Var Compensator) system to reject arbitrary disturbances relative degree from its output channels (used for shunt compensation to maintain bus voltage magnitude). [32] presents a compound disturbanceobserver-based MPC scheme applied for a wheeled mobile robot which aims 85 at compensating the slowly varying disturbances affecting the control inputs. A similar approach for trajectory tracking of a small helicopter is considered in [33] where the disturbance observer ensures the system's robustness against constant wind gusts. Furthermore, there are many works related to disturbance rejection for USVs using observer-based robust control. The authors in 90 [34, 35, 36] employ a nonlinear observer within a backstepping technique to design a trajectory tracking robust controller of the underactuated ship. How- 
ever, the constraints coming from physical limitations are not considered. To the best of the authors knowledge, there are very few studies using disturbance observer-based MPC to ensure the robustness of surface vehicle in case of uncertainties. [18, 37] present obstacle and collision avoidance in the presence of external disturbances while tracking a reference path, but they only take one ship into account which operates in a simple environment. For that reason, practical application of safe navigation in a complex coastal environment for multi-surface vehicles under uncertainties remains an open problem.

\subsection{Contributions}

Motivated by all the observations above and the results of [38], the paper introduces enhancements in the distributed motion planning for safe navigation of multiple surface vehicles in the presence of external disturbance in the Trondheim fjord complying with the COLREGS rules. Specifically, the contributions of this paper are:

1. generates LOS (Line-of-Sight) guidance system via a graph-based method, e.g., through the RRT* (optimal Rapidly-Exploring Random Tree) algorithm;

2. consider on-off barrier functions which guarantee the necessary connectivity distance for information exchange among the agents as well as activate the associated repulsive potential for static and dynamic obstacles;

3. consider a NDO (Nonlinear Disturbance Observer) to reject the disturbances from the ocean that may lead to undesirable performance for the ships;

4. integrate the above ingredients in a distributed NDO-NMPC - based algorithm with a threefold purpose: i) track the RRT* - based feasible path through LOS guidance system, ii) activate the constraints in the view range $\rfloor^{1}$ of the agent for on-line collision avoidance complying with the

\footnotetext{
${ }^{1}$ As required by COLREGS, all ships shall maintain a proper radar lookout (has a view of up to several kilometers if there are no physical obstructions) to obtain early warning of risk of collision.
} 
COLREGs rules and iii) exchange information for connectivity maintenance;

5. validation of the proposed algorithm through simulations over a real benchmark for the safe navigation of ships in the Trondheim fjord.

\subsection{Outline}

The paper is organized as follows: Section 2 presents the multi-agent dynamical models and the on-off repulsive potential constructions. Section 3 presents the distributed motion planning algorithm for connectivity maintenance and COLREGS compliance. Section 4 shows the simulation results over a real benchmark. Section 5 draws the conclusions and presents the future work.

\section{Prerequisites}

This paper is inspired by the practical application of collision-free motion planning of unmanned surface vessels traveling between harbors in the Trondheim fjord, Norway. Fig. 1 illustrates the operating region of the ships which need to navigate to the desired haven while avoiding the shore or small islands and other vessels (moving obstacles) while simultaneously complying with the COLREGS rules ${ }^{2}$ (see Apendix A).

As a benchmark we have used the Automatic Identification Systems (AIS) to provide real numerical data related to the position and velocity of ships, as they navigate between harbors.

In order to efficiently describe the non-convex feasible region for the dynamical (mobile) agent, we briefly recall here the system dynamics and various notions which involve polyhedral sets and repulsive potential constructions.

\footnotetext{
${ }^{2}$ http:/ /astat.autonomous-ship.org/
} 


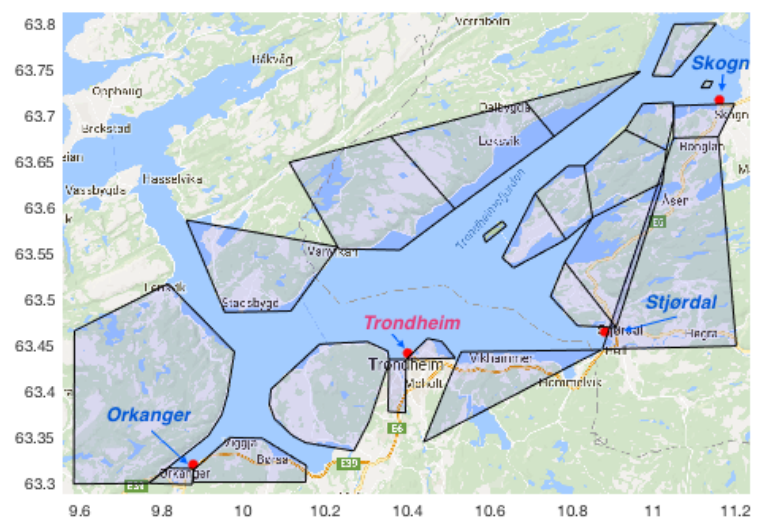

Figure 1: Map of Trodheim obtained from real data: feasible space and considered forbidden cells as in 4.

\subsection{Multi-autonomous surface vehicles dynamics}

Let us consider a set $\mathcal{V}$ including $N$ agents, $\mathcal{V}=\{1, \ldots, N\}, N \geq 2$, operating in a two-dimensional work space. The nonlinear dynamics of agent $i \in \mathcal{V}$ under external disturbances (wind, wave and current) are described in [39]:

$$
\dot{x}_{i}=f_{i}\left(x_{i}(t), \mathbf{u}_{i}(t), w_{i}(t)\right)=\left\{\begin{aligned}
\dot{\eta}_{i} & =R_{i}\left(\psi_{i}\right) v_{i} \\
M_{i} \dot{v}_{i} & =-C_{i}\left(v_{i}\right) v_{i}-D_{i} v_{i}+\mathbf{u}_{i}+w_{i}
\end{aligned}\right.
$$

where the state vector, $\mathbf{x}_{i}=\left[\begin{array}{ll}\eta_{i} & v_{i}\end{array}\right]^{\top} \in \mathbb{R}^{6}$ includes the vector $\eta_{i}=\left[\begin{array}{ll}p_{i} & \psi_{i}\end{array}\right]^{\top} \in$ $\mathbb{R}^{3}$ with $p_{i}=\left[\begin{array}{ll}x_{i} & y_{i}\end{array}\right]^{\top}$, the system position and $\psi_{i}$, the yaw angle in the inertial frame. It also includes vector $v_{i}=\left[\begin{array}{lll}u_{i} & v_{i} & r_{i}\end{array}\right]^{\top} \in \mathbb{R}^{3}$ describing the surge, sway and yaw rates. The input vector, $\mathbf{u}_{i} \in \mathbb{R}^{3}$, with $\mathbf{u}_{i}=\left[\begin{array}{lll}\mathcal{T}_{u_{i}} & 0 & \mathcal{T}_{r_{i}}\end{array}\right]^{\top}$ contains the surge thrust and rudder deflection. The addictive disturbance $w_{i}=\left[\begin{array}{lll}w_{u_{i}} & 0 & w_{r_{i}}\end{array}\right]^{\top} \in \mathbb{R}^{3}$ is not a pure Gaussian noise but rather the output of such a noise (after it passed through a nonlinear filter), and is bounded by $\left\|w_{i}\right\| \leq \varrho_{i}$ and accounts for the ocean environment ${ }^{3}$ Also, in $11, R_{i}\left(\psi_{i}\right), M_{i}$,

\footnotetext{
${ }^{3}$ I.e., wind, wave, ocean current affect control input of the ship.
} 
$C_{i}\left(v_{i}\right)$ and $D_{i} \in \mathbb{R}^{3 \times 3}$ are the rotation, mass, Coriolis and damping matrices, respectively ${ }^{4}$.

\subsection{On-off repulsive potential field framework}

Let us first introduce the following Logistic Regression function, which was first applied for population growth studies [41] and is commonly used in machine learning algorithms for two-class classification:

$$
F(x)=\frac{L}{1+e^{\left(x-x_{0}\right) \beta}},
$$

where $x_{0}$ is the value of the sigmoid's midpoint, $L$ is the curve's maximum value and $\beta$, the steepness of the curve. Note that a negative or positive value of $\beta$ will determine the properties of the on-off barrier function which we will define hereinafter. Fig. 2 shows the on-off barrier function for $L=1, x_{0}=100$ and varying $\beta$.

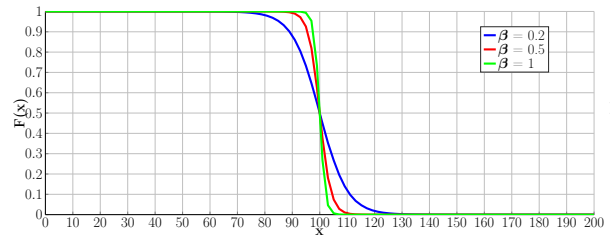

(a) $\beta>0$.

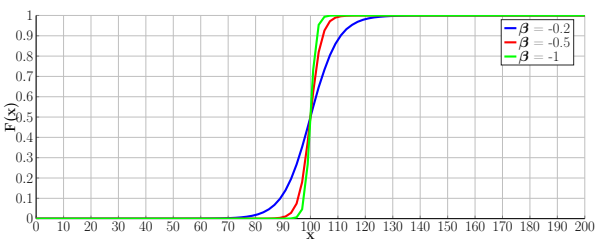

(b) $\beta<0$.

Figure 2: On-off barrier function with different $\beta$ as in 2 .

In this work we design on-off repulsive potential fileds as a combination between on-off barrier functions and repulsive potential fields for static and moving obstacles in order to activate and deactivate their influence in the agent's view range.

\footnotetext{
${ }^{4}$ Note that in model [1], the side-slip is neglected as presented in [40], therefore yaw and course are the same.
} 
Assume that agent $i \in \mathcal{V}$ can perceive its neighbors (other agents) and the forbidden cells (fixed obstacles 5 ) as well as transmit information to its neighbors within the range $\rho_{i}\left(p_{i}, r_{\rho}^{i}\right)$, where $r_{\rho}^{i} \in \mathbb{R}>0$ is the radius of the ball centered in $p_{i} \in \mathbb{R}^{2}$, the current position of agent $i$.

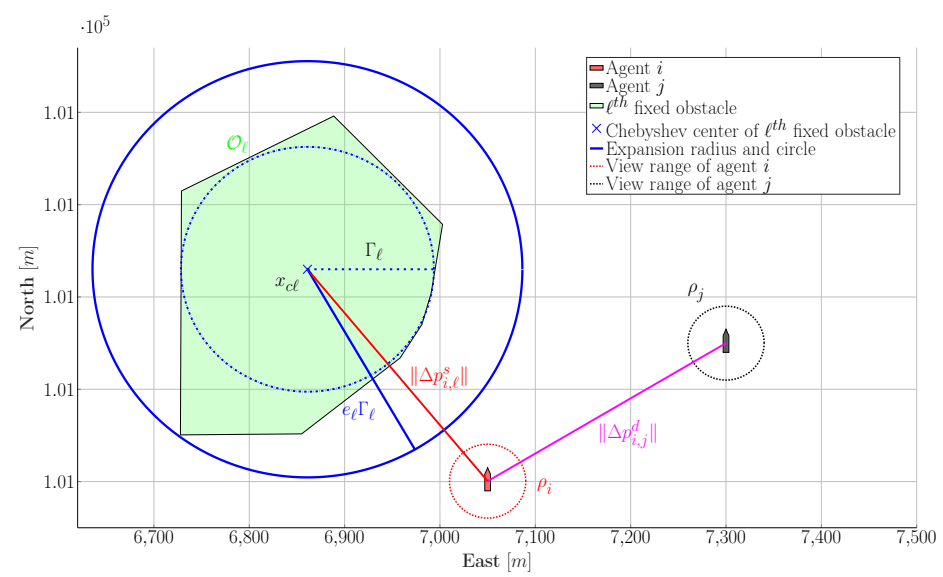

Figure 3: Description of the safe distance of the $i^{\text {th }}$ agent w.r.t. the $\ell^{\text {th }}$ cell and $j^{\text {th }}$ agent.

For simplicity, the range $\rho_{i}\left(p_{i}, r_{\rho}^{i}\right)$ is considered hereinafter simultaneously as the view range (i.e., the zone where the ship can detect all the object) and the communication range (i.e., the area where the ships can exchange information with each other).

The safe distance between the agent and the fixed obstacles, $D_{s}^{i, \ell}$, is given by:

$$
D_{S}^{i, \ell}=e_{i} \Gamma_{\ell}+\rho_{i}
$$

where $e_{i}$ is a scaling parameter, $\Gamma_{\ell}$ is the radius of the circle defined from the Chebyshev center of the $\ell^{\text {th }}$ forbidden cell and $\rho_{i}$ is the range of the ship. Moreover, the range $\rho_{i}\left(p_{i}, r_{\rho}^{i}\right)$ of the $i^{\text {th }}$ agent is also considered as a safe distance of itself with respect to the other agents/moving obstacles (see Fig. 3).

\footnotetext{
${ }^{5}$ The fixed obstacles such as islands and shorelines are a priori known from maps. The ship's position in relation to the obstacles is known using Global Navigation Satellite Systems.
} 


\subsubsection{Repulsive potential field of static obstacles}

Let us define the static obstacles (islands, shore and the like) by a union of forbidden polytopic convex regions as:

$$
\mathrm{O}=\bigcup_{\ell=1}^{N_{\text {cell }}} \mathcal{O}_{\ell} .
$$

where $\mathcal{O}_{\ell}=\left\{p_{i} \in \mathbb{R}^{2} \mid a_{k}^{\ell} p_{i} \leq b_{k}^{\ell}, k=1, \ldots, n_{h}\right\}$, with $a_{k}^{\ell} \in \mathbb{R}^{1 \times 2}, b_{k}^{\ell} \in \mathbb{R}, n_{h}^{\ell}$ is the number of half-spaces describing $\mathcal{O}_{\ell}$ and $N_{\text {cell }}$ is the number of forbidden polytopic regions.

For each bounded polyhedral $\mathcal{O}_{\ell}$, we consider the piecewise linear function $\sqrt{6}$ as in [42]:

$$
\gamma_{\ell}\left(p_{i}\right)=\sum_{k=1}^{n_{h}^{\ell}}\left(a_{k}^{\ell} p_{i}-b_{k}^{\ell}+\left|a_{k}^{\ell} p_{i}-b_{k}^{\ell}\right|\right) .
$$

Using (5), we define $\mathrm{S}$ as a union of repulsive potentials of the fixed obstacles as:

$$
\mathbf{S}=\sum_{i=1}^{N_{\text {cell }}} S_{\ell}^{\mathrm{fix}}\left(\gamma_{\ell}\left(p_{i}\right)\right),
$$

with $S_{\ell}^{\text {fix }}\left(\gamma_{\ell}\left(p_{i}\right)\right)$ given as:

$$
S_{\ell}^{\mathrm{fix}}\left(\gamma_{\ell}\left(p_{i}\right)\right)=\frac{c_{1 \ell}}{\left(c_{2 \ell}+\gamma_{\ell}\left(p_{i}\right)\right)^{2}}
$$

where $c_{1 \ell}$ and $c_{2 \ell}$ are positive parameters representing the strength and effect ranges of repulsive potential.

Hence, the on-off repulsive potential of the fixed obstacle, $\Omega_{\text {cell }}$, is considered as the repulsive potentials of the static obstacles described as in (6) will be activated/deactivated if the on-off barrier function from (2) is considered as follows:

$$
\Omega_{\text {cell }}=\sum_{\ell=1}^{N_{\text {cell }}} F_{i, \ell}\left(\Delta p_{i, \ell}^{s}, D_{s}^{i, \ell}\right) S_{\ell}^{\mathrm{fix}}\left(p_{i}\right)
$$

\footnotetext{
${ }^{6}$ The function 5 is zero inside the convex set $\mathcal{O}_{\ell}$ defined as in 4 and non-zero with a value
} which grows piecewise linearly as the distance from the set $\mathcal{O}_{\ell}$ increases. 
with $S_{\ell}^{\text {fix }}\left(p_{i}\right)$ given in $(7), F_{i, \ell}($.$) defined as an on-off barrier function and based$ on the safety distance $\left(D_{S}^{i, \ell}\right)$ between agent $i$ and the forbidden obstacle $\ell$ :

$$
F_{i, \ell}(.)=\frac{L}{1+e^{\left(\left\|\Delta p_{i, \ell}^{s}\right\|-D_{s}^{i, \ell}\right) \beta}},
$$

Proposition 1. If a set $\mathbb{S}$ of $N_{\text {cell }} \in \mathbb{N}$ representing static repulsive potential fields eq. (6) are only partially activated by the agent's view range through on-off barrier functions as defined in eq. (8), then a null-potential field7 value which implies a local minima can be excluded.

\subsubsection{Repulsive potential field of agents/moving obstacles}

Hereinafter, we represent the moving obstacles and the safety regions around the agents as polytopic regions parametrized in function of their current position. Therefore, let us define $\mathcal{P}_{i \backslash\{j\}},(i, j \in \mathcal{V}, i \neq j)$ as the safety region of agent $i$ that agent $j$ must avoid:

$$
\mathcal{P}_{i \backslash\{j\}}=\left\{p_{i}, p_{j} \in \mathbb{R}^{2}: a_{m}^{i}\left(p_{j}-p_{i}\right) \leq b_{m}^{i}, m=1, \ldots, n_{h}^{j}\right\},
$$

where $p_{i}, p_{j}$ are the current positions of agents $i, j ; a_{m}^{i} \in \mathbb{R}^{1 \times 2}, b_{m}^{i} \in \mathbb{R}, n_{h}^{i}$ is the number of half-spaces describing $\mathcal{P}_{i \backslash\{j\}}$.

Next, we define a piecewise linear function similar to (5) for the safety region of agent $i$ :

$$
\theta_{i, j}\left(p_{i}, p_{j}\right)=\sum_{k=1}^{n_{h}^{i}}\left(a_{m}^{i}\left(p_{j}-p_{i}\right)-b_{m}^{i}+\left|a_{m}^{i}\left(p_{j}-p_{i}\right)-b_{m}^{i}\right|\right) .
$$

Similarly, using (11), we define the repulsive potentials which take into account the shape of the agent $i$ 's safety regions:

$$
S_{i, j}^{\mathrm{mov}}\left(\theta_{i, j}\left(p_{i}, p_{j}\right)\right)=\frac{c_{1 j}}{\left(c_{2 j}+\theta_{i, j}\left(p_{i}, p_{j}\right)\right)^{2}} .
$$

\footnotetext{
${ }^{7}$ Where the various potential components cancel each other.
} 
Consequently, in order to activate or deactivate the repulsive potentials of agents or moving obstacles established as in (12), the on-off barrier function from (2) will be integrated into the construction of the repulsive potential to define for the on-off repulsive potential of the moving obstacles:

$$
\Omega_{\mathrm{mov}}=\sum_{j=1, j \neq i}^{N} F_{i, j}(.) S_{i, j}^{\mathrm{mov}}\left(p_{i}, p_{j}\right),
$$

where $S_{i, j}^{\operatorname{mov}}\left(p_{i}, p_{j}\right)$ is given in (12) and $F_{i, j}\left(\Delta p_{i, j}^{d}, \rho_{i}\right)$ defined as follows:

$$
F_{i, j}(.)=\frac{L}{1+e^{\left(\left\|\Delta p_{i, j}^{d}\right\|-\rho_{i}\right) \beta}}
$$

with $\rho_{i}$ defined as $i^{\text {th }}$ agent's view range.

Note that $\beta$ in (9) and (14) is chosen positive to ensure that if the distance between agent and fixed/moving obstacles is greater than the safety distance between them, then the value of the on-off barrier function goes to zero thus inactivating the repulsive potential and, otherwise, and coupled with $L=1$ it conserves the repulsive potential's value.

\subsection{Connectivity maintenance condition}

We consider here that the information known to agents in a group (trajectories, velocities, and the like) can only be exchanged with those which are inside the communication range.

Let us consider the undirected graph $\mathcal{G}=(\mathcal{V}, \mathcal{E})$ where $\mathcal{V}$ is the set of nodes representing all agents and $\mathcal{E}=\{(i, j) \in \mathcal{V} \times \mathcal{V}: i \leftrightarrow j\}$ is the set of edges of the graph. The adjacency matrix $\mathcal{A}=\left[a_{i j}\right]$ indicates if a pair of nodes are adjacent or not in the graph hence satisfying the property:

$$
a_{i j}= \begin{cases}1, & \text { if }(i, j) \in \mathcal{E}, \\ 0, & \text { otherwise } .\end{cases}
$$

Consequently, the condition of connectivity maintenance for information exchange among agent $i$ and its neighbors $\left(\mathcal{N}_{i}\right)$ is defined as follows:

$$
\mathcal{N}_{i}=\left\{j \in \mathcal{V} \backslash\{i\}:\left\|\Delta p_{i, j}\right\|<\min \left(\rho_{i}, \rho_{j}\right)\right\},
$$


where, $\rho_{i}$ and $\rho_{j}$ are the view range of agents $i$ and $j$ in (3).

Next, for any edge between two nodes of set $\mathcal{N}_{i}$, we define an on-off barrier function as in (2) whose value is always zero within the interval $\left[0, \min \left(\rho_{i}, \rho_{j}\right)\right)$ :

$$
M_{i, j}\left(p_{i}, p_{j}\right)=\frac{L_{i j}}{1+e^{\left(\left\|\Delta p_{i, j}^{d}\right\|-\min \left(\rho_{i}, \rho_{j}\right)\right) \beta_{i j}}} .
$$

Note that in this case, $\beta_{i j}<0$ and $L_{i j}$ has a high value such that the distance among the agents of $\mathcal{N}_{i}$ does not exceed the $\min \left(\rho_{i}, \rho_{j}\right)$.

Using the above construction we define a so called "connectivity maintenance function" of agent $i$ with its neighbors $\mathcal{N}_{i}$ :

$$
\mathcal{M}_{i}\left(p_{i}, p_{j}\right)=\sum_{j \in \mathcal{N}_{i}} M_{i, j}\left(p_{i}, p_{j}\right)
$$

\subsection{Path generation with LOS guidance}

Usually for the control part is easier to have a priori defined a path which can be then tracked on-line. In here, RRT* is applied for generating an off-line optimal collision-free path in the workspace for a known initial state $\left(x_{\text {init }}\right)$ and set of fixed obstacles $(\mathrm{O})$. The general idea of the RRT* algorithm $([43,44])$ is to initialize a tree $\mathscr{T}$ from an initial vertex $\left(x_{\text {init }}\right)$ and to bias growth towards unexplored regions of the state space randomly. In the process of exploring the tree, shortest paths are generated while verifying the collision-free condition with respect to the fixed obstacles after each iteration until reaching a new state close enough to the desired target. The feasible path is established based on the connection of shortest paths.

LOS algorithms have been used for autonomous ships navigation by [39]. The feasible path obtained from RRT* is a set of waypoints $\left(x_{p}, y_{p}\right)$ in the horizontal plane.

For simplicity, assuming that the along-track error, $x_{e}$, is zero. Thus, crosstrack error $\left(y_{e}\right)$ for the surface vehicle position as in Fig. 4 is given by:

$$
\left[\begin{array}{l}
0 \\
y_{e}
\end{array}\right]=\left[\begin{array}{cc}
\cos \left(\delta_{p}(\alpha)\right) & -\sin \left(\delta_{p}(\alpha)\right) \\
\sin \left(\delta_{p}(\alpha)\right) & \cos \left(\delta_{p}(\alpha)\right)
\end{array}\right]^{\top}\left[\begin{array}{c}
x-x_{d}(\alpha) \\
y-y_{d}(\alpha)
\end{array}\right]
$$




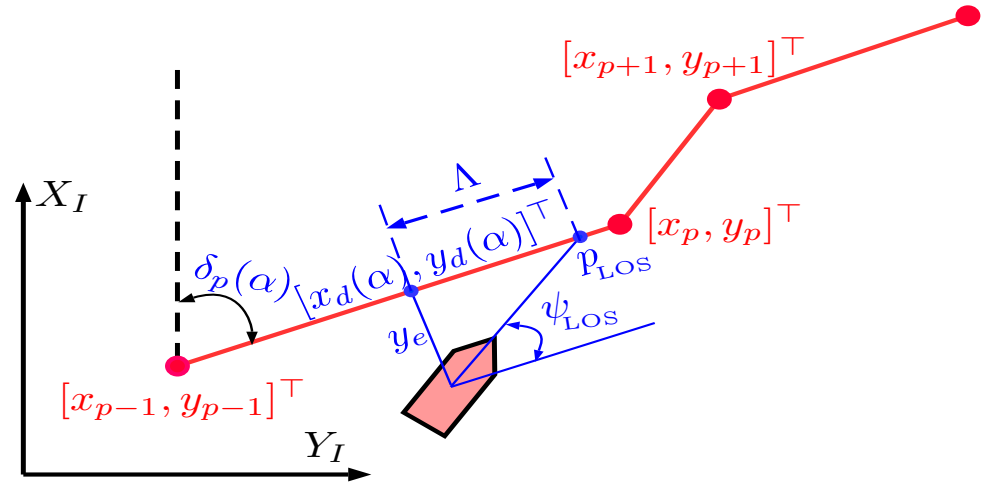

Figure 4: LOS guidance for a feasible path.

where $x_{d}(\alpha), y_{d}(\alpha)$ are parametrized by $\alpha$ as:

$$
\begin{aligned}
& x_{d}(\alpha)=x_{p}+\alpha \cos \left(\delta_{p}(\alpha)\right), \\
& y_{d}(\alpha)=y_{p}+\alpha \sin \left(\delta_{p}(\alpha)\right),
\end{aligned}
$$

where $\delta_{p}(\alpha)$ and $\left(x_{p}, y_{p}\right)$ are the orientation and the initial position of the path initialized from way-point $p$, with $\alpha$ parametrization variable is a scalar.

The LOS angle, $\psi_{\text {LOS }}$ (as can be seen in Fig. 44, is calculated based on the cross-track error $y_{e}$ and the lookahead distance $\Lambda$, expressed as:

$$
\psi_{L O S}=-\frac{y_{e}}{\sqrt{y_{e}^{2}+\Lambda^{2}}}
$$

The angle, $\psi_{\mathrm{LOS}} 8$ is considered as a reference state of the heading angle $\psi$ of surface vehicles to ensures convergence of the ship's position to a feasible path generated by RRT*.

\footnotetext{
${ }^{8}$ Note that, since locally $\Lambda$ corresponds to the inverse proportional gain [45], the convergence to the path depends on the value of $\Lambda$. A low value means faster convergence than a larger one, but with a large overshoot.
} 


\section{Motion planning for connectivity maintenance with COLREGS compli- ance in the presence of external disturbances}

This section presents the motion planning algorithm for multi-surface vehicles connectivity maintenance. The motion planning strategy is illustrated via the block diagram in Fig. 5. From a map of the Trondheim fjord, the ground represented by static obstacles and the coordinates of the harbors can be acquired as seen in Fig. 1. A collision-free path, and LOS guidance system can then be generated off-line from the starting harbor to the desired destination based on a set of waypoints yielded by the RRT* algorithm. NDO-based NMPC, a feedback control scheme in which an optimization problem is solved on-line at each time step, will ensure collision avoidance for dynamic obstacles and robustness in the presence of the external disturbances. If there are no static or dynamic obstacles, the NDO-based NMPC will try to re-establish path following of the path obtained from RRT*

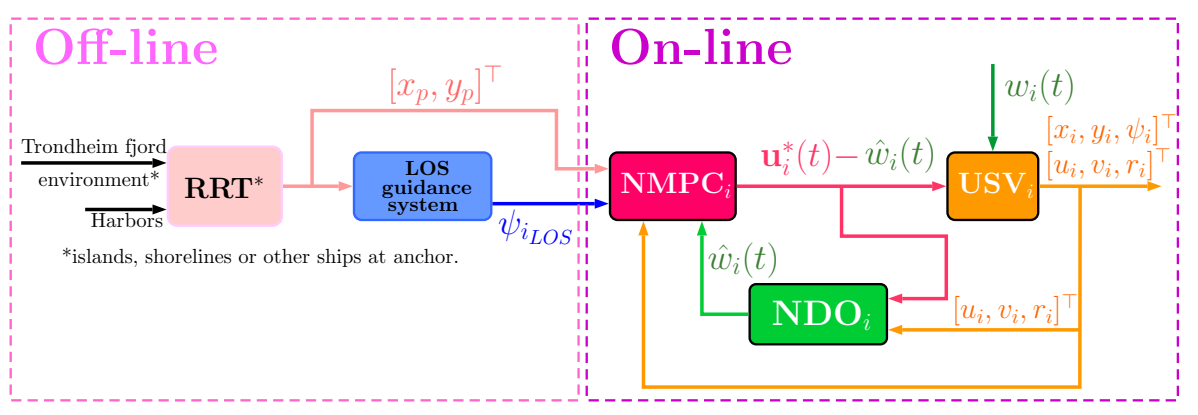

Figure 5: Motion planning strategy.

\subsection{NDO design for unknown time-variant disturbance}

The unknown varying external disturbances (e.g., ocean currents) impact directly the control inputs of vessels leading to bad performance. Consequently, the nonlinear disturbance observer will provide an estimate of the disturbance, and its estimation is fed back to the NMPC solver. Subsequently, a disturbance 
observer is presented for a general nonlinear dynamic which will be later used for the estimation of the disturbance vector $w_{i}$ affecting the i-th agent.

A fundamental idea of disturbance observer in NMPC scheme is to estimate the unknown disturbance $w_{i}$ by an estimate $\hat{w}_{i}$ such that $\left|\hat{w}_{i}-w_{i}\right| \rightarrow 0$. In fact, the acceleration, $\dot{v}_{i}$, is not available in many robotic manipulators, and it is also difficult to construct the acceleration signal from the velocity signal by differentiation due to measurement noise. Therefore, we first need to define the $q_{i}=M_{i} v_{i}$ as the auxiliary variable and its estimation $\hat{q}_{i}$. Consequently, the estimated error, $q_{i e}$ is described as below:

$$
q_{i e}=\hat{q}_{i}-q_{i}
$$

From the (1), The time derivative of $\hat{q}_{i}$ can be rewritten as:

$$
\dot{q}_{i}=-C_{i}\left(v_{i}\right) v_{i}-D_{i}\left(v_{i}\right) v_{i}+\mathbf{u}_{i}
$$

As the results of [34, 46], the time derivative of auxiliary variable's estimation, $\hat{q}_{i}$ is defined as follows:

$$
\dot{\hat{q}}_{i}=L\left(-C_{i}\left(v_{i}\right) v_{i}-D_{i}\left(v_{i}\right) v_{i}+\mathbf{u}_{i}-h\left(q_{i e}\right)\right),
$$

where $L=\operatorname{diag}\left\{c_{1}, c_{2}, c_{3}\right\}>0$, are the gains of the NDO, and $h\left(q_{i e}\right) \in \mathbb{R}^{3}$ is a design vector to be determined.

Consequently, the time derivative of estimated error between auxiliary variable, $q_{i}$ and its estimation, $\hat{q}_{i}$ described as:

$$
\begin{aligned}
\dot{q}_{i e} & =\dot{\hat{q}}_{i}-\dot{q}_{i} \\
& =\left(L-\mathbf{I}_{3}\right)\left[-C_{i}\left(v_{i}\right) v_{i}-D_{i}\left(v_{i}\right) v_{i}+\mathbf{u}_{i}\right]-\operatorname{Lh}\left(q_{i e}\right),
\end{aligned}
$$

where $\mathbf{I}_{3}$ is the identity matrix with dimension related to the state space vector of $v_{i}$.

To ensure that 25) converges to zero, 25) has to satisfy two conditions:

1. $L=\mathbf{I}_{3}$,

2. The designed function vector converges towards zero, i.e., $h\left(q_{i e}\right) \rightarrow 0$. 


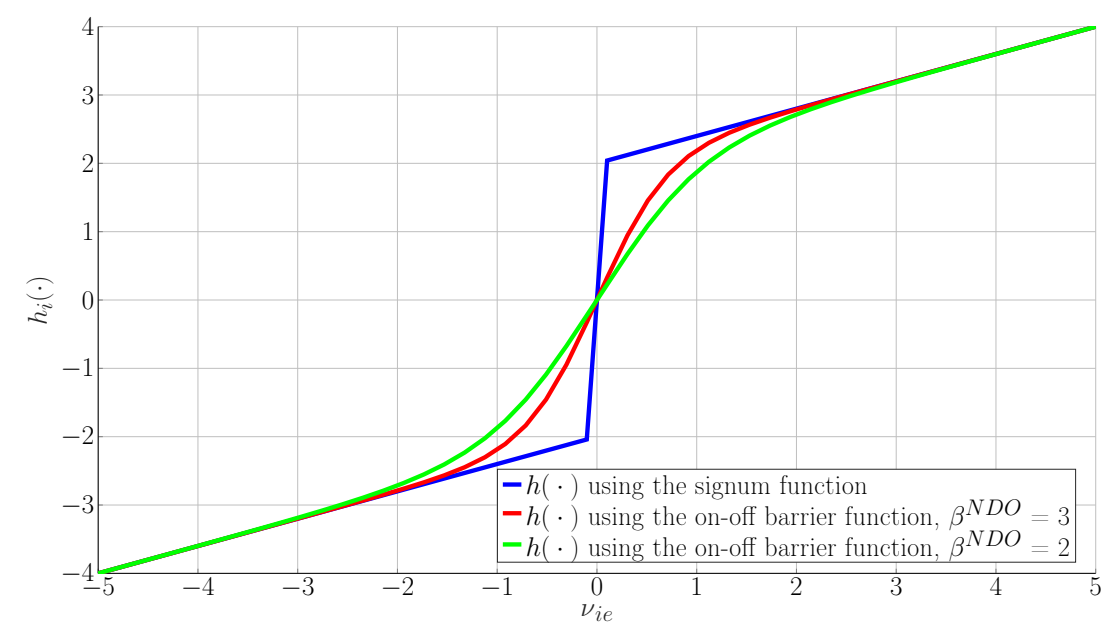

Figure 6: The comparison of designed function $h_{i}(\cdot)$ 26 between on-off barrier function (with the various value of $\beta^{N D O}>0$ ) and signum function.

We propose the use of on-off barrier function with continuous nature in order to design $h\left(q_{i e}\right)$ based on the estimated error, $q_{i e}$ in 22). Hence, the design function, $h\left(q_{i e}\right)$, is described as follows:

$$
h\left(q_{i e}\right)=K_{1 i}\left(q_{i e}\right)+K_{2 i}\left|q_{i e}\right| \mathcal{J}\left(q_{i e}\right)+d_{i} \mathcal{J}_{i}\left(q_{i e}\right),
$$

where $\mathcal{J}_{i}\left(q_{i e}\right)=\frac{e^{\beta_{i}^{N D O}} M_{i} v_{i e}}{\mathbf{1}+e^{\beta D O_{i}} q_{i e}}-\frac{e^{-\beta_{i}^{N D O} q_{i e}}}{\mathbf{1}+e^{-\beta_{i}^{N D O}} q_{i e}} \in \mathbb{R}^{3}$ is a vector whose dimension depends on vector $q_{i e} \in \mathbb{R}^{3} ; K_{1 i}=\operatorname{diag}\left\{K_{1 i}^{1}, K_{1 i}^{2}, K_{1 i}^{3}\right\}, K_{2 i}=\operatorname{diag}\left\{K_{2 i}^{1}, K_{2 i}^{2}, K_{2 i}^{3}\right\}$ and $d_{i}=\operatorname{diag}\left\{d_{i}^{1}, d_{i}^{2}, d_{i}^{3}\right\}$ positive semi-definite matrices.

Remark 1. In 447 , the authors employed signum function $\left(\mathcal{J}_{i}\left(q_{i e}\right)=\frac{\left|q_{i e}\right|}{q_{i e}}\right)$ to design function $h\left(q_{i e}\right)$. However, a discontinuity at zero is the main drawback which comes from the property of the signum function. Since design function $h\left(q_{i e}\right)$ considers the estimation error of the real disturbance, a discontinuity at zero means that the convergence of this error toward zero will be indefinite.

Fig. 6 compares the proposed function $h_{i}(\cdot)$ with two different values for $\beta^{N D O}$ with the original signum function as in 26). 
With $h_{i}(\cdot)$ as in (26), (24) can be written as:

$$
\begin{aligned}
\dot{\hat{q}}_{i}= & -C_{i}\left(v_{i}\right) v_{i}-D_{i}\left(v_{i}\right) v_{i}+\mathbf{u}_{i} \\
& -K_{1 i}\left(q_{i e}\right)-K_{2 i}\left|q_{i e}\right| \mathcal{J}_{i}\left(q_{i e}\right)-d_{i} \mathcal{J}_{i}\left(q_{i e}\right) .
\end{aligned}
$$

The estimated disturbance, $\hat{w}_{i}$ will be given by:

$$
\begin{aligned}
\hat{w}_{i} & =\dot{\hat{q}}_{i}+C_{i}\left(v_{i}\right) v_{i}+D_{i}\left(v_{i}\right) v_{i}-\mathbf{u}_{i} \\
& =-K_{1 i}\left(q_{i e}\right)-K_{2 i}\left|q_{i e}\right| \mathcal{J}\left(q_{i e}\right)-d_{i} \mathcal{J}\left(q_{i e}\right)=h\left(q_{i e}\right) .
\end{aligned}
$$

The following proposition is introduced to highlight the convergence of estimated disturbances toward the real disturbances.

Proposition 2. Consider the additive disturbances in (1). Given the NDO as in Eq. (27)-28, its estimate $\hat{w}$ can asymptotically track the input disturbances $w$. In other words, $\|\hat{w}-w\|_{\infty} \rightarrow 0$.

Proof. Consider the following Lyapunov function

$$
V_{i}=\frac{1}{2}\left(q_{i e}\right)^{\top}\left(q_{i e}\right) .
$$

The time derivative of the Lyapunov function is

$$
\begin{aligned}
\dot{V}_{i} & =\left(q_{i e}\right)^{\top}\left(\dot{q}_{i e}\right)=\left(q_{i e}\right)^{\top}\left(\dot{\hat{q}}_{i}-M_{i} \dot{v}_{i}\right) \\
& =\left(q_{i e}\right)^{\top}\left[-K_{1 i}\left(q_{i e}\right)-K_{2 i}\left|q_{i e}\right| \mathcal{J}_{i}\left(q_{i e}\right)-d_{i} \mathcal{J}_{i}\left(q_{i e}\right)\right] \\
& \leq-\left(q_{i e}\right)^{\top} K_{1 i}\left(q_{i e}\right)-\left(q_{i e}\right)^{\top} K_{2 i}\left|q_{i e}\right| \mathcal{J}_{i}\left(q_{i e}\right) \\
& \leq-2 K_{1 i_{\text {min }}} V_{i}-2 K_{2 i_{\text {min }}} V_{i} \\
& \leq 0
\end{aligned}
$$

where $K_{1 i_{\text {min }}}=\min \left\{K_{1 i}^{1}, K_{1 i}^{2}, K_{1 i}^{3}\right\}$ and $K_{2 i_{\text {min }}}=\min \left\{K_{2 i}^{1}, K_{2 i}^{2}, K_{2 i}^{3}\right\}$.

The estimated error $q_{i e}$ will converge to zero (i.e., $q_{i e} \rightarrow 0$ ) due to 30 . 
Combining (1) and (28), the disturbance error $w_{i e}$ is rewritten as follows:

$$
\begin{aligned}
w_{i e} & =\hat{w}_{i}-w_{i} \\
& =-K_{1 i}\left(q_{i e}\right)-K_{2 i}\left|q_{i e}\right| h_{i}\left(q_{i e}\right)-d_{i} h_{i}\left(q_{i e}\right)-M_{i} v_{i}-C_{i} v_{i}-D_{i} v_{i}+\mathbf{u}_{i} \\
& =\dot{\hat{q}}_{i}-\dot{q}_{i}=\dot{q}_{i e},
\end{aligned}
$$

where $h_{i}\left(v_{i}\right)$ was defined in 26. Since $v_{i e}$ is the velocity error (surge, sway and yaw rate), if $v_{i e}$ converges to zero, $\dot{v}_{i e}$ (acceleration's error) will converge to zero also. Hence, using the NDO as in 27)-28, the observer $\hat{w}_{i}$ will converge towards the external disturbance $w_{i}$.

\subsection{NMPC - based distributed motion planning with disturbance compensation}

NMPC - based distributed approach of multi-agent systems has an operational mechanism based on information exchange between an agent and its neighbors to converge towards a standard solution. Figure. (7) illustrates the distributed motion planning in the context of this paper, if the connectivity among agents is guaranteed, each controller of the agent receives information from the others and updates its actions to reach a consensus. The disturbances acting on the agents are compensated by using the estimates obtained with the nonlinear observer.

Let us summarize in the following the necessary ingredients for formulating the motion planning with disturbance compensation algorithm:

1. to obtain more flexible behavior, the lookahead distance, $\Lambda$ from 21 is time-varying and optimized to obtain accelerated convergence and little overshoot.

2. to satisfy collision avoidance with the ground and comply to rules 8 and 13 of the COLREGS with moving obstacles we add the repulsive potential constructions from (8), (13) in an NMPC optimization problem which will be activated only in the view range of the agents;

3. to comply with rules 14 and 15 of the COLREGS we add a slack variable in the cost to give priority to a negative rate of change of yaw moment, i.e., turning to starboard side as in [18]. 


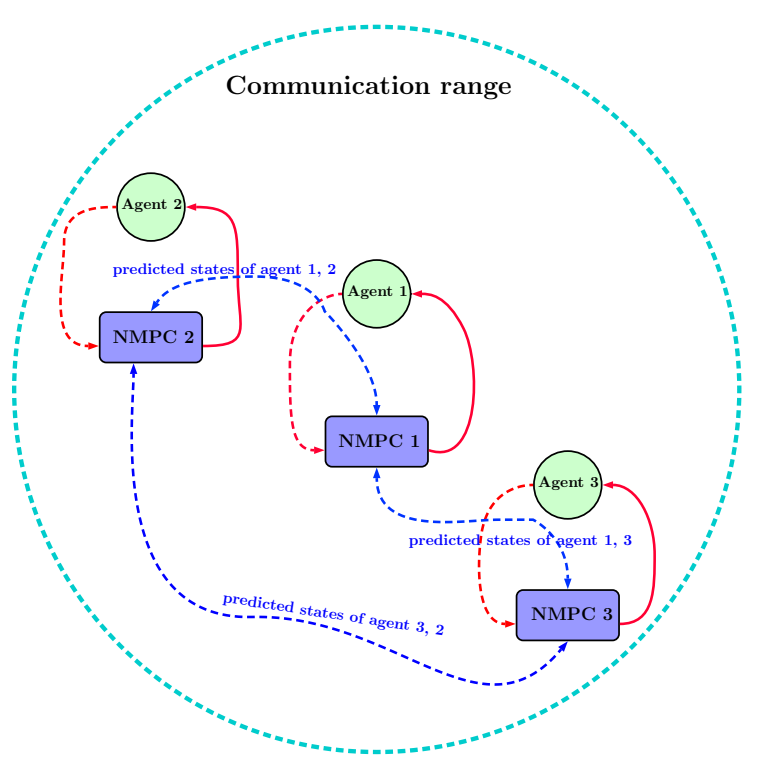

Figure 7: Distributed motion planning architecture.

4. to ensure the agents' connectivity we add in the optimization problem the connectivity function given in 18;

5. to reject the unknown disturbance from the current affecting the control system of the agents, the output of the NDO as in (27)-(28) is introduced as a compensation signal;

6. the implementation is done in a distributed fashion, i.e., the agents exchange information only with those inside their communication range.

The nominal system of $i^{\text {th }}$ agent is defined from Eq. (1) by neglecting the disturbances, i.e., $w_{i}(t) \equiv 0$, and given by

$$
\dot{\bar{x}}_{i}=f_{i}\left(\bar{x}_{i}(t), \overline{\mathbf{u}}_{i}(t), 0\right)
$$

For each nominal system 32 we solve a finite horizon open-loop OCP (optimal control problem) at time $t$, using the measured state $x_{i}(t)$ over the prediction horizon $T_{p}$ :

$$
\min _{\overline{\mathbf{u}}_{i}(\cdot)} \int_{t}^{t+T_{p}}\left[\mathcal{L}_{i}\left(\bar{x}_{i}(\tau), \bar{x}_{j}(\tau), \overline{\mathbf{u}}_{i}(\tau)\right)\right] d \tau+E\left(\bar{x}_{i}\left(t+T_{p}\right)\right),
$$


subject to:

$$
\begin{aligned}
& \dot{\bar{x}}_{i}=f_{i}\left(\bar{x}_{i}, \overline{\mathbf{u}}_{i}, 0\right), \bar{x}_{i}(t)=x_{i}(t), \\
& \overline{\mathbf{u}}_{i}(\tau) \in \mathcal{U}_{i}, \\
& \Lambda \leq \Lambda(\tau) \leq \bar{\Lambda}, \\
& \epsilon_{i}(\tau)>0, \\
& \dot{\mathcal{T}}_{r_{i}}(\tau)-\epsilon_{i}(\tau)<0, \\
& \bar{x}_{i}(\tau) \in \mathcal{X}_{i}, \\
& \bar{x}_{j}(\tau) \in \mathcal{X}_{j}, \forall \tau \in\left[t, t+T_{p}\right] .
\end{aligned}
$$

The stage cost $\mathcal{L}_{i}($.$) has the following expression:$

$$
\begin{aligned}
\mathcal{L}_{i}(.)= & \left\|\bar{x}_{i}(\tau)-x_{i, r e f}(\tau)\right\|_{Q_{i}}^{2}+\left\|\overline{\mathbf{u}}_{i}(\tau)\right\|_{R_{i}}^{2}+\left\|\dot{\mathbf{u}}_{i}(\tau)\right\|_{\Delta R_{i}}^{2}+\Omega_{\text {cell }}\left(\bar{x}_{i}(\tau)\right) \\
& +\Omega_{\operatorname{mov}}\left(\bar{x}_{i}(\tau), \bar{x}_{j}(\tau)\right)+\mathcal{M}_{i}\left(\bar{x}_{i}(\tau), \bar{x}_{j}(\tau)\right)+\left\|\epsilon_{i}(t)\right\|_{S_{i}}^{2} .
\end{aligned}
$$

and the terminal cost is defined as:

$$
E\left(\bar{x}_{i}\left(t+T_{p}\right)\right)=\left\|\bar{x}_{i}\left(t+T_{p}\right)-x_{i, r e f}\right\|_{P_{i}}^{2}
$$

In (34a), $f_{i}(\cdot, \cdot)$ is presented in (1), $\bar{x}_{i}(\tau), \overline{\mathbf{u}}_{i}(\tau)$ are the predicted states and inputs while $\overline{\mathbf{u}}_{i}($.$) represents the predicted input trajectory along the prediction$ horizon $T_{p}$. In the cost per stage $35, \dot{\mathbf{u}}_{i}(\tau)$ denotes the predicted input variations, $x_{i, r e f}$ is the reference trajectory, $\Omega_{\text {cell }}$ and $\Omega_{\text {mov }}$ are the on-off barrier repulsive potential for collision avoidance given in (8) and (13) while $\mathcal{M}_{i}$ penalises the connectivity maintenance between agent $i$ and its neighbors as defined in (18) and the last term $\epsilon_{i}$ is the slack variable prioritizing a certain yaw orientation. $Q_{i}, R_{i}, P_{i}$ and $S_{i}$ are (semi)-positive definite weighting matrices of appropriate dimensions. The constraint of lookahead distance, $\Lambda(\tau)$ is presented in Eq. $34 \mathrm{c}$. Constraint $34 \mathrm{~d}$ ) is tightened by the positive slack variable, $\epsilon_{i}(34 \mathrm{e})$ in order to prioritize the negative rate of change of the yaw moment. This forces the vessel to turn to starboard in any situation, hence complying with the rules 13, 14 and 15 of COLREGS (see Appendix). Rule 8 is also obeyed through the use of the repulsive potential in 13 . 
In (36), $x_{i, r e f}$ is the collision-free path generated using RRT $^{*}$ and detailed in the forthcoming section.

At each sampling instance, each agent solves its OCP (33) and yields the minimizing control sequence for the nominal system (32) over the interval $\left[t, t+T_{p}\right]$. Then, only the first sample of the control input will be combined with the estimated disturbance in a closed-loop to yield a compound control as follows:

$$
\mathbf{u}_{i}(t)=\mathbf{u}_{i}^{*}(t)-\hat{w}_{i}(t),
$$

where $\mathbf{u}_{i}^{*}(t)$ is the output of the minimization problem (33), (34).

From (37), agents compensate external disturbances in real-time and obtain a predicted collision-free trajectory $\bar{x}_{i}(\tau)$ through the use of repulsive potential 325 fields constructed for fixed and moving obstacles. The information (predicted trajectory) of agent $i$ will be transmitted to its neighbor $j \in \mathcal{N}_{i}$ whenever the connectivity between them holds. Then the agent $j$ with the information updated from agent $i$ will solve its own OCP (33). This procedure will continue until agent $i \in \mathcal{V}$ solves its own problem (33) and is repeated at next sampling instance.

Note that the repulsive potentials of static obstacles (7) and the moving obstacle/other ships (12) are taken into account over the prediction horizon for collision avoidance, and is called repeatedly, for each time instant $\tau$. However, they are disabled due to combining with on-off barrier function as in 9) and (14) (it has a "0" value if nothing is in the ship's view range). The repulsive potential will be activated via on-off barrier function (it has a "1" value if the moving obstacle/other ships or the fixed obstacles are in ship's view range). This approach has shown effectiveness when the potential field changes constantly via on-off barrier function thus allowing the vessel to avoid getting trapped inside a local minima [38]. 


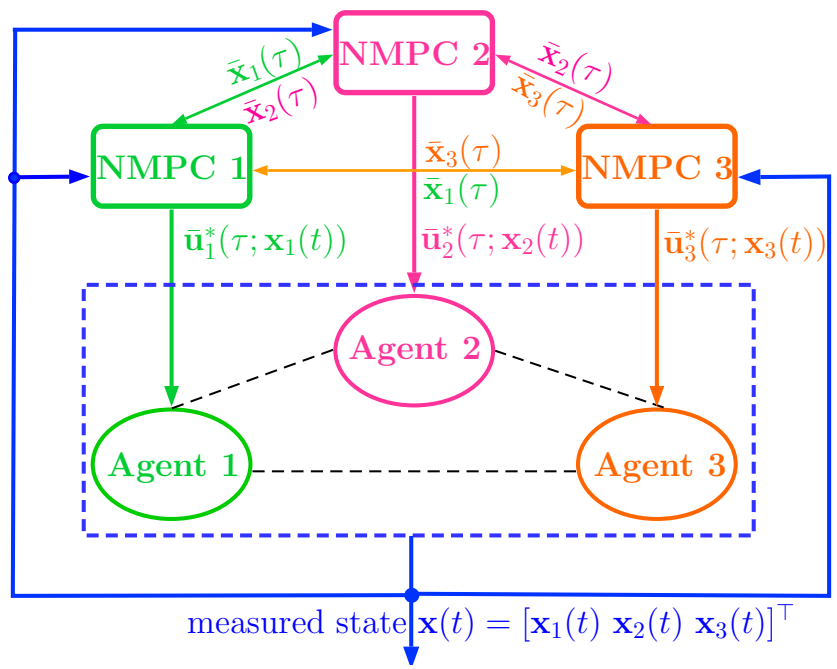

Figure 8: Parallel distributed NMPC architecture.

Note that the distributed NMPC scheme employed is a parallel architecture with the aim of improving the closed-loop performance for USVs (compared with the sequential architecture) [48]. More specifically, the separate NMPC controllers are evaluated based on a new measured state (as can be seen $x(t)$ in Fig. 8) and broadcast their information (i.e., predicted states, for example, $\bar{x}_{1}(\tau), \bar{x}_{2}(\tau)$ and $\bar{x}_{3}(\tau)$ in Fig. 8) to their neigbors if they stay interior of the communication range in parallel at the same sampling time. Fig. 8 illustrates the communication topology of the distributed control framework for three agents.

The details are presented in Algorithm 1 .

Several remarks are as follows:

Remark 2. Only the nominal system (32) is utilized in OCP.

Remark 3. The initial state of NDO, $\hat{q}_{i}(\tau)(27)$, has to be provided.

Remark 4. Regarding the rate of change of yaw moment, $\dot{\mathcal{T}}_{r_{i}}$, there are two possible 355 cases: i) $\dot{\mathcal{T}}_{r_{i}}>0$ (i.e., ship will turn to port side), ii) $\dot{\mathcal{T}}_{r_{i}}<0$, (i.e., ship will turn to starboard). 
The slack variable weight should be carefully chosen so that $\dot{\mathcal{T}}_{r_{i}}$ can only be a very small positive number but still satisfies constraint (34e) while the slack variable, $\epsilon_{i}$, is always a small positive number as in (34d). Therefore, this case $\left(\dot{\mathcal{T}}_{r_{i}}>0\right)$ is improbable. In other words, the case $\left(\dot{\mathcal{T}}_{r_{i}}<0\right)$ is strongly encouraged.

Remark 5. The prediction horizon should be chosen large enough to ensure cover of the agent's view range or at least assure that the agent has time enough to avoid the imminent collision. Conversely, the prediction horizon cannot be chosen arbitrarily large due to numerical and computational issues.

The procedure of information exchange among the agents in Algorithm 1 is based on [49]. Collision-free motion planning with the rejection of external disturbances through NDO is proposed by the authors and described in the following:

Algorithm 1 Information exchange procedure and collision avoidance of the NMPC-based distributed motion planning with the rejection of external disturbances through NDO.

Require: Consider the set of agents $\mathcal{V}$ with the nominal model (1) with $w_{i}=$ 0 ), the set of repulsive potentials of the forbidden cells (7) and of the agents (12), the on-off barrier function as in (9) and (14), the safe distance $D_{s}^{i, \ell}$, the ranges of agents $i, j \in \mathcal{V}$, and set of waypoints obtained off-line through RRT*.

1: $\tau \in\left[t, t+T_{p}\right]$;

2: Initialize the NDO as in (27)-(28);

3: for $i=1: N$ do

4: Update the information of its neighbors $\left(\mathcal{N}_{i}\right)$;

5: $\quad$ if $\left\|\Delta p_{i, \ell}^{s}\right\|>D_{s}^{i, \ell}$ and $\left\|\Delta p_{i, j}^{d}\right\|>\min \left(\rho_{i}, \rho_{j}\right)$ then

6: $\quad$ inactivate repulsive potential of fixed obstacles and agent $j$ in (35);

7: $\quad$ end if 
8: $\quad$ if $\left\|\Delta p_{i, \ell}^{s}\right\|>D_{s}^{i, \ell}$ and $\left\|\Delta p_{i, j}^{d}\right\| \leq \min \left(\rho_{i}, \rho_{j}\right)$ then

9: $\quad$ inactivate repulsive potential of fixed obstacles and activate repulsive potential of agent $j$ in (35);

10: end if

11: $\quad$ if $\left\|\Delta p_{i, \ell}^{s}\right\| \leq D_{s}^{i, \ell}$ and $\left\|\Delta p_{i, j}^{d}\right\|>\min \left(\rho_{i}, \rho_{j}\right)$ then

12: $\quad$ activate repulsive potential of fixed obstacles and inactivate repulsive potential of agent $j$ in 35 ;

13: end if

14: $\quad$ if $\left\|\Delta p_{i, \ell}^{s}\right\| \leq D_{s}^{i, \ell}$ and $\left\|\Delta p_{i, j}^{d}\right\| \leq \min \left(\rho_{i}, \rho_{j}\right)$ then

15: $\quad$ activate repulsive potential of fixed obstacles and agent $j$ in 35;

16: end if

17: end for

18: Agent $i$ solves OCP 33 and obtains the predicted collision-free trajectory $\bar{x}_{i}(\tau)$;

19: Transmit $\bar{x}_{i}(\tau)$ to its neighbors $j \in \mathcal{N}_{i}$;

20: Apply only the first sample of the compound control sequence as in 37) to both the nominal system and NDO over the interval $\left[t, t+T_{p}\right]$;

21: Continue to the next sampling instance;

22: RETURN step 1; 


\section{SIMULATIONS}

370 terizes a real ship at a scale of 1:70 (thus, various parameters which appear later like view range, ship length, surge bound, are scaled proportionally). Since we aim to test our algorithm on a realistic benchmark (using real movement data, as given by the Automatic Identification Systems (AIS) ), we scale all the in-

(e.g., the distance between harbors used in simulation is 70 times less than the real one).

Consider a set of $N=3$ underactuated ships with the dynamical model (1) with the length overall (LOA) is $1.255[\mathrm{~m}]$, the matrice ${ }^{9}{ }^{9}, M, D$ and $B$ taken from [50]:

$$
\begin{gathered}
R_{i}=\left[\begin{array}{ccc}
\cos \psi_{i} & -\sin \psi_{i} & 0 \\
\sin \psi_{i} & \cos \psi_{i} & 0 \\
0 & 0 & 1
\end{array}\right], M_{i}=\left[\begin{array}{ccc}
25.8 & 0 & 0 \\
0 & 33.8 & 1.0115 \\
0 & 1.0115 & 2.76
\end{array}\right], \\
D_{i}=\left[\begin{array}{ccc}
0.9257 & 0 & 0 \\
0 & 2.8909 & -0.2601 \\
0 & -0.2601 & 0.5
\end{array}\right]
\end{gathered}
$$

These vessels are simulated to navigate in the Trondheim fjord (Norway). Fig. 1 1illustrates the operating region of the ships which need to navigate to different harbors while maintaining connectivity and avoiding the shore or small islands and other vessels. The AIS is used to provide real numerical data related to the position and velocity of ships, as well as time of navigation between harbors.

The three agents in the group need to maintain the connectivity among them while traveling from Orkanger to Trondheim harbor (for situation 1) and

\footnotetext{
${ }^{9}$ For simplicity, the Coriolis matrix is neglected.
} 
Stjordal harbor (for situation 2) and avoid fixed obstacles and a mobile obstacle which has the dynamical model described in (1).

The number of cells considered from partitioning the map as in Fig. 1 is $390 \quad N_{\text {cell }}=22$.

Since the view-range is used both to receive (i.e., detect) obstacles' information and transmit signals to other ships, the view-range of the ships 3 with $\rho_{i}=70[\mathrm{~m}]$ is assumed. The scaling coefficient is $e_{i} \in[1.2,1.5]$.

The steepness of the repulsive potential defined in (9) and (14) is given by $\beta_{i}=1$. The steepness of connectivity maintenance function $(18), \beta_{i j}$ and curve's maximum value, $L_{i j}$ used as in (17) are $\beta_{12}=\beta_{13}=\beta_{23}=-0.002$ and $L_{12}=L_{13}=L_{23}=1000$. Other parameters of the NMPC optimization problem in (33) are chosen as follows: the weighting matrices $Q_{i}=0.1 I_{6}, R_{i}=0.1 I_{2}$, $P_{i}=\left[\begin{array}{lllllll}0.5 I_{2} & 0_{2} & 0_{2} ; 0_{2} & I_{2} & 0_{2} ; 0_{2} & 0_{2} & I_{2}\end{array}\right], S_{i}=10^{-6}$, we consider a conservatively chosen prediction horizon $T_{p}=6 s$ in order to asses less than ideal conditions (decisions have to be made when the other ships/obstacles are already very close) and the sampling time is 2 s.

The design parameters of the NDO in (27) and (28) are chosen as follows $K_{1 i}=\operatorname{diag}\{0.2,0,0.2\}, K_{2 i}=\operatorname{diag}\{0.025,0,0.025\}=d_{i}, \lambda_{i}=1$ and ${ }_{405} \beta_{i}^{N D O}=1$.

We consider the surge velocity of agent 1 to be the highest $\left(u_{1} \in[-0.2,0.6]\right.$ $[\mathrm{m} / \mathrm{s}])$; the surge velocities of agent 2 and 3 equal $\left(u_{2}=u_{3} \in[-0.2,0.4]\right.$ $[\mathrm{m} / \mathrm{s}])$ and less than the surge velocity of agent 1 . The yaw angles' constraint is $[-\pi, \pi]$. We consider constraints on the actuation force $\mathcal{T}_{u_{1}}=\mathcal{T}_{u_{2}}=\mathcal{T}_{u_{3}} \in$ ${ }_{410}[-2,2][\mathrm{N}]$ and on the yaw moment $\mathcal{T}_{r_{1}}=\mathcal{T}_{r_{2}}=\mathcal{T}_{r_{3}} \in[-0.2,0.2][\mathrm{Nm}]$. The lookahead distance is taken in the interval, $\Lambda \in[2 \mathrm{LOA}, 10 \mathrm{LOA}]$ where LOA is the maximum length of a ship's hull, 1.255 [m] as stated in [50].

The simulations are done using IPOPT solver and CASADI [51] toolkit in Matlab R2016a on a computer with the following configuration: Intel Core i74790CPU, 3.60GHz, 8GB RAM. 


\subsection{Scenario 1}

This scenario presents a comparison of the proposed method in this paper and the approach of [52] in the context of COLREGS compliance, in particular, rule 8 , in the absence of uncertainties. The approach of [52] first considers the Euclidean distance as a collision avoidance constraint among the agents, and then proposes a penalty term ensuring that any alteration of course and speed must be significant enough to clear the approaching ships. The two conditions will be taken into account over a prediction horizon.

The method proposed by [52] penalizes the slow alterations of yaw rate and surge velocity of ship 1 , as can be seen in Fig. 9 . At time step $k=82$, the yaw rate (drawn in dashed blue) is altered rapidly. The decrease in yaw rate implies that ship 1 turns starboard. The yaw rate in red color changes gradually, and changes earlier than the one in dashed blue at time step $k=78$, increase in yaw rate means that ship 1 turns port. Also, the Euclidean distance between ship 1 and 2, which is implemented by Algorithm 1 is greater than the safe threshold whereas the relative distance between the two ships of Breivik's approach approaches the safe limit in a critical moment as depicted in Fig. 10.

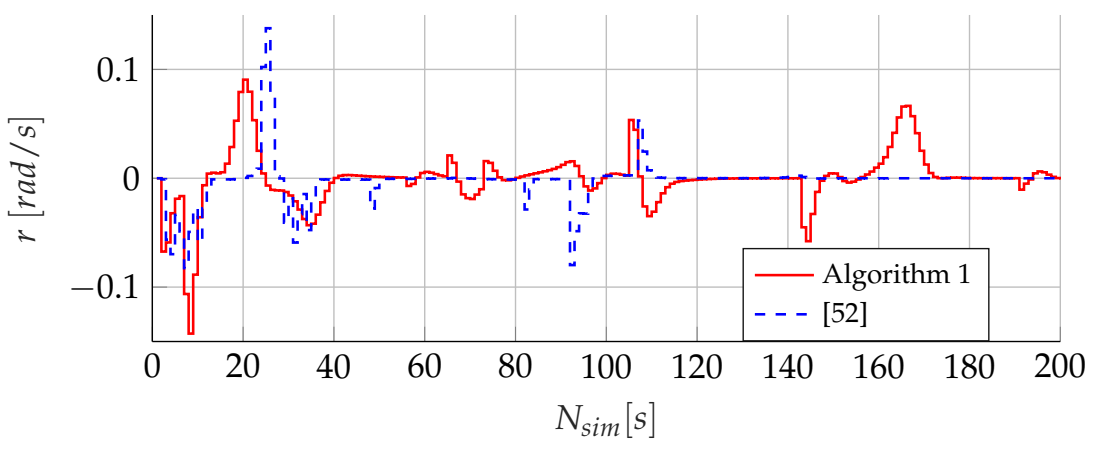

Figure 9: Comparison of yaw rates of two approaches. 


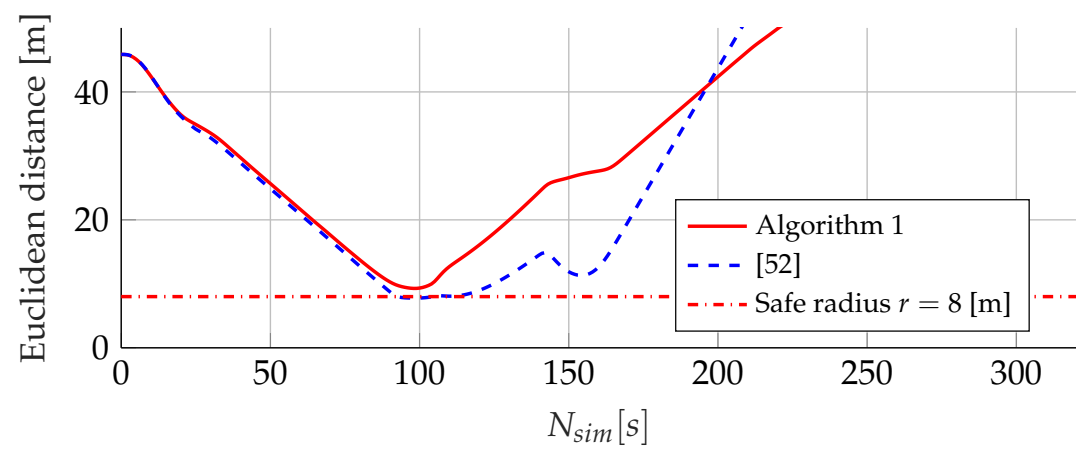

Figure 10: Comparison of Euclidean distance of two approaches.

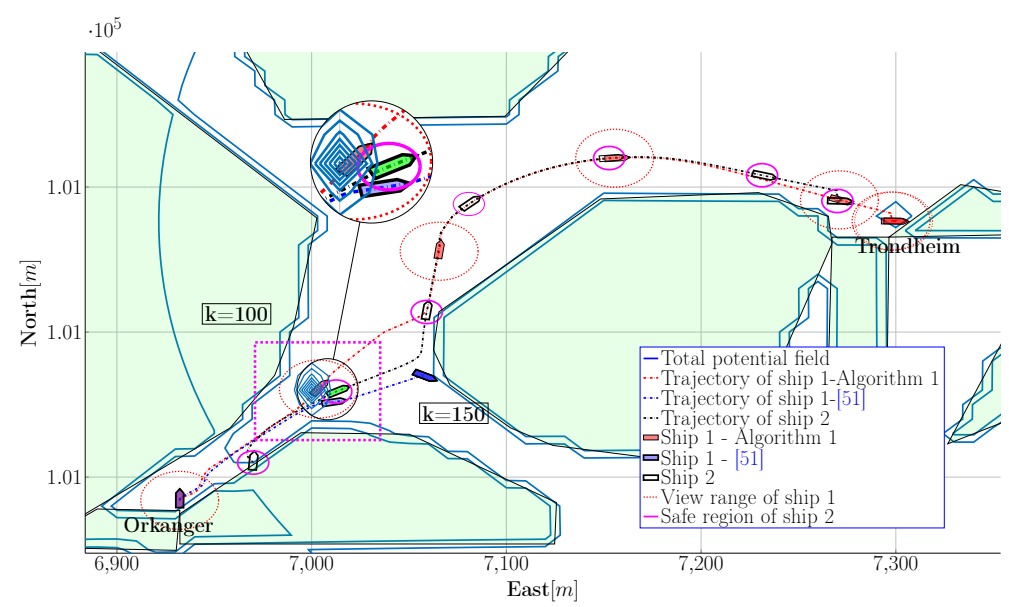

Figure 11: Motion planning of two USVs while traveling from Orkanger to Trondheim harbor with COLREGS compliance - rules 8.

Fig. 11, shows the actual motion of two USVs while traveling from Orkanger to Trondheim harbors, while complying with rule 8 of COLREGS. The ship 1 (in blue) for which we used the algorithm proposed by [52] is trapped at time step $k=150$ where it becomes cornered within a "deadlock" region due to simultaneously guaranteeing the safe distance with ship 2 and avoiding the coast. Meanwhile, ship 1 (in red), for which we use our proposed potential field approach for moving obstacle, reaches the Trondheim harbor in a timely 

for the three ships/moving obstacle will not be illustrated in order to have less cluttered illustrations.

\subsection{Scenario 2}

Scenario 1 evaluates the validity of motion planning for connectivity maintenance in a group of three vessels in the course of going to desired harbors, while maintaining collision avoidance, and complying with the COLREG rules with dynamic obstacles under LOS guidance in the absence of disturbance.

\subsubsection{Situation 1}

Fig. 12 shows the off-line collision-free path generated using the RRT* algorithm after 1500 iterations. Fig. 13 depicts the actual motion of the three vessels

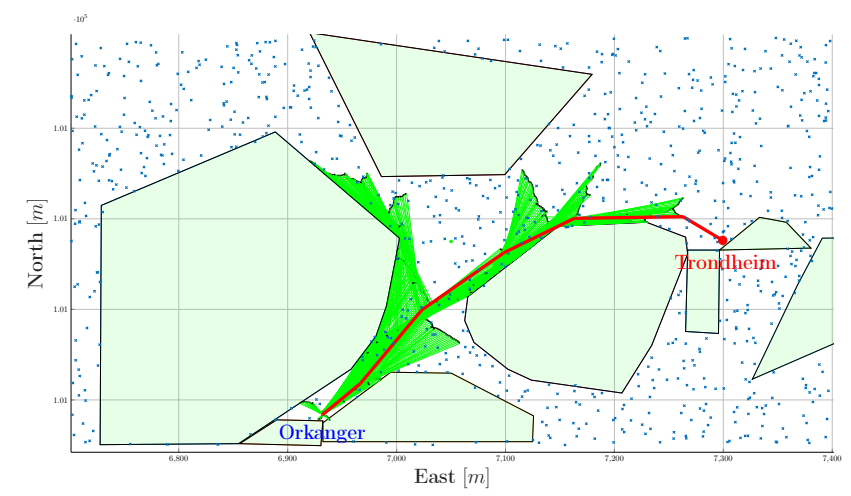

Figure 12: Feasible collision-free path from Orkanger to Trondheim harbor.

traveling from Orkanger to Trondheim harbor. In the same figure, we show the group of agents at 7 different time instances as they are changing their formation configuration, for instance due to the moving obstacle motion illustrated in black. Fig. 17 shows that the connectivity condition is maintained. 


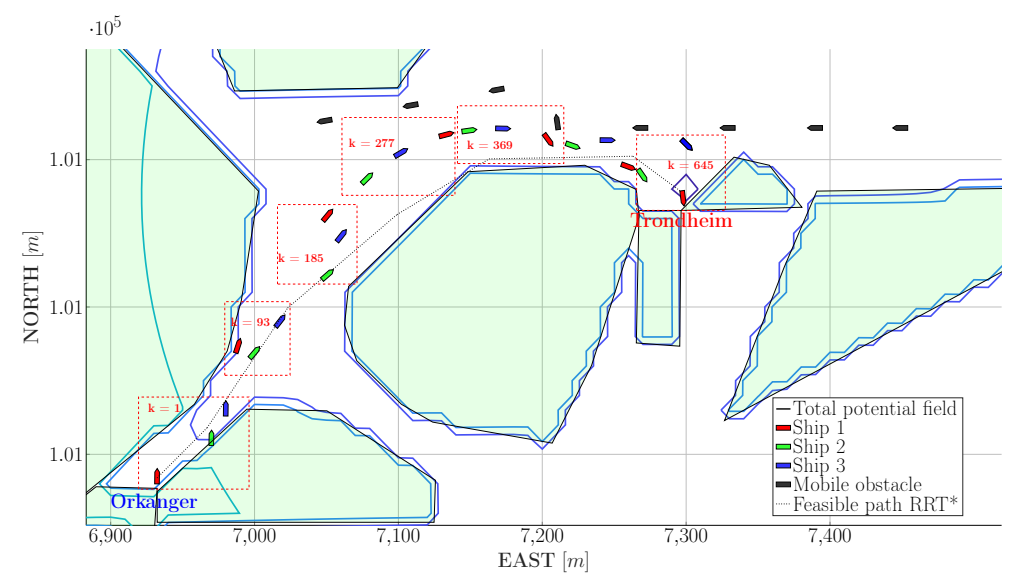

Figure 13: Connectivity maintenance of the 3 USVs while traveling from Orkanger to Trondheim harbor with COLREGS compliance - rules 13 and 14.
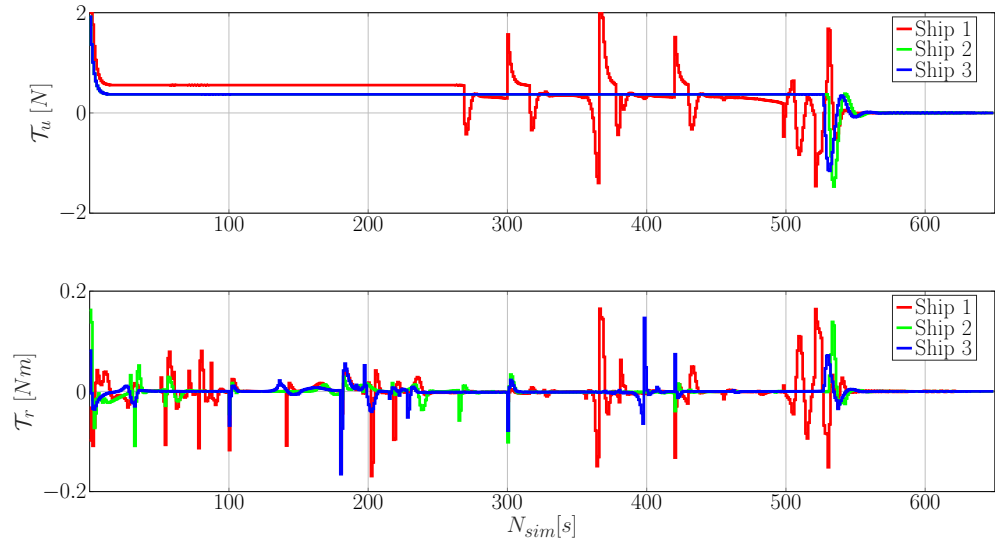

Figure 14: Control inputs of the three vessels in situation 1.

At time instance $k=93$, the ship 1 (in red) is overtaking on the port side of the second and third ship, hence satisfying the COLREGS rule 13 - the overtaking situation. This can also be observed in Fig. 16 and 15 where ship 2 (in green) and 3 (in blue) are being overtaken, not changing their course and speed until step $k=135$. 


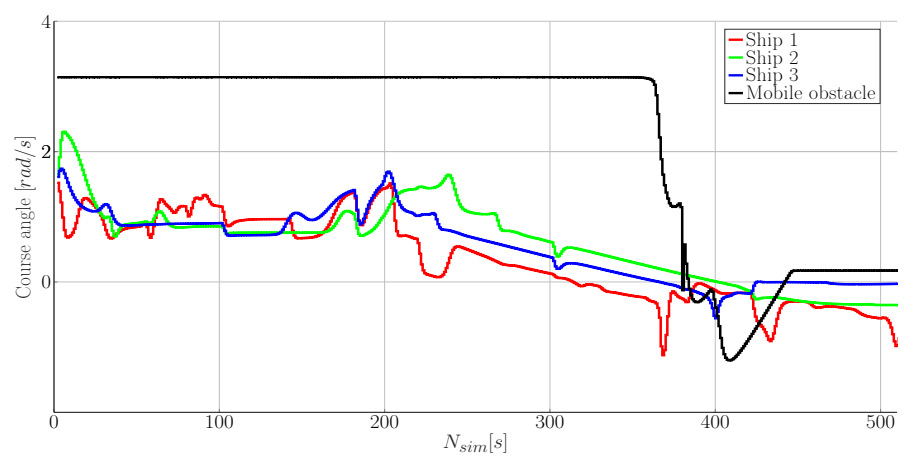

Figure 15: Course angles of the three vessels and obstacle ship in situation 1.

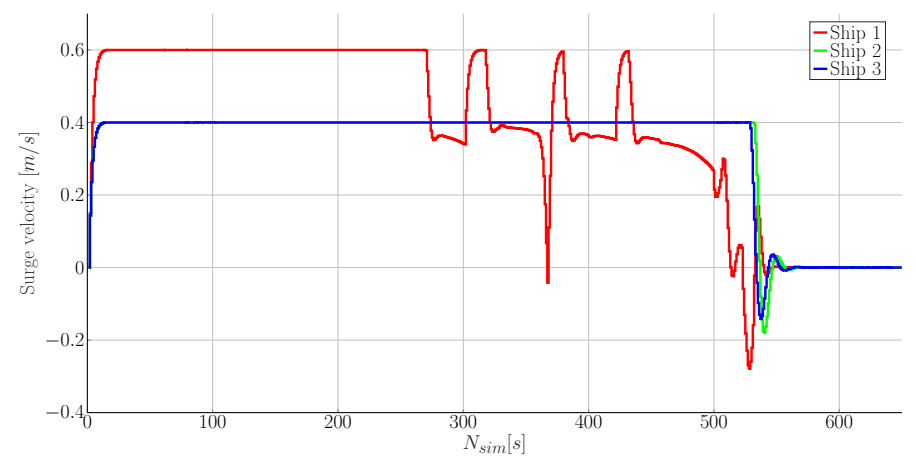

Figure 16: Surge velocities of the three vessels in situation 1.

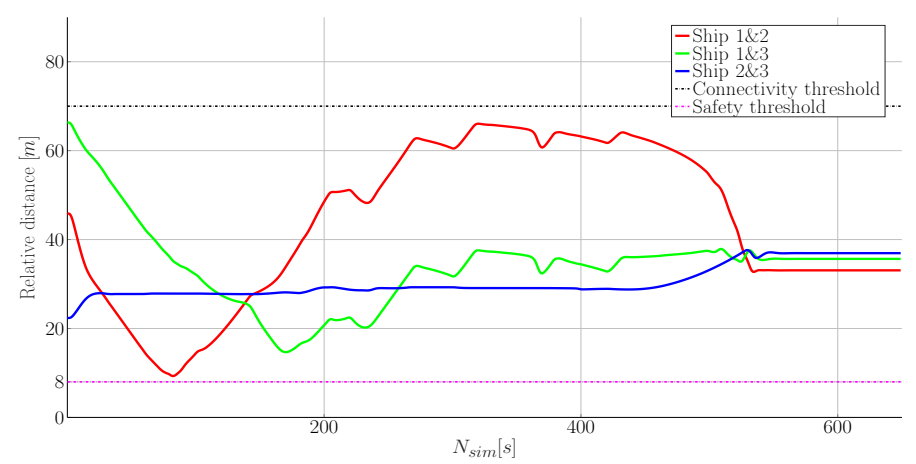

Figure 17: Relative distances of the three agents in situation 1.

In time interval $k \in[365,400]$ the COLREGS rule 14 concerning head-on 
collisions between ships and/or mobile obstacles, becomes active. The first ship in the group (in red) and the mobile obstacle start changing their course angles to turn to their starboard (right side) at time step $k=365$ (Fig. 15), then the course angle of the third ship of the group (in blue) has a significant increase at time step $k=400$ (see also Fig. 15). Note that if the course angle of the ship is increasing, then the vessel is steered to its starboard and vice-versa.

Fig. 14 depicts the control inputs corresponding to surge thrust and rudder deflection of the three vessels.

\subsubsection{Situation 2}

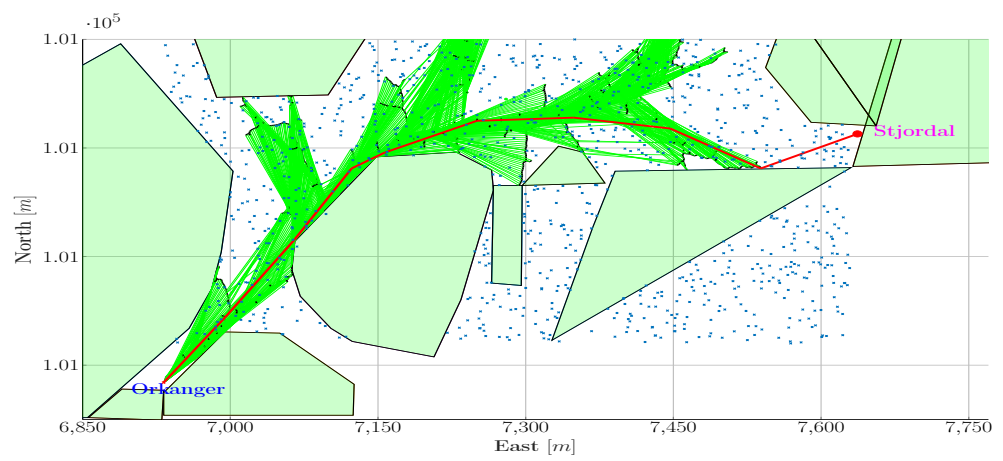

Figure 18: Feasible collision-free path Orkanger to Stjordal harbor.

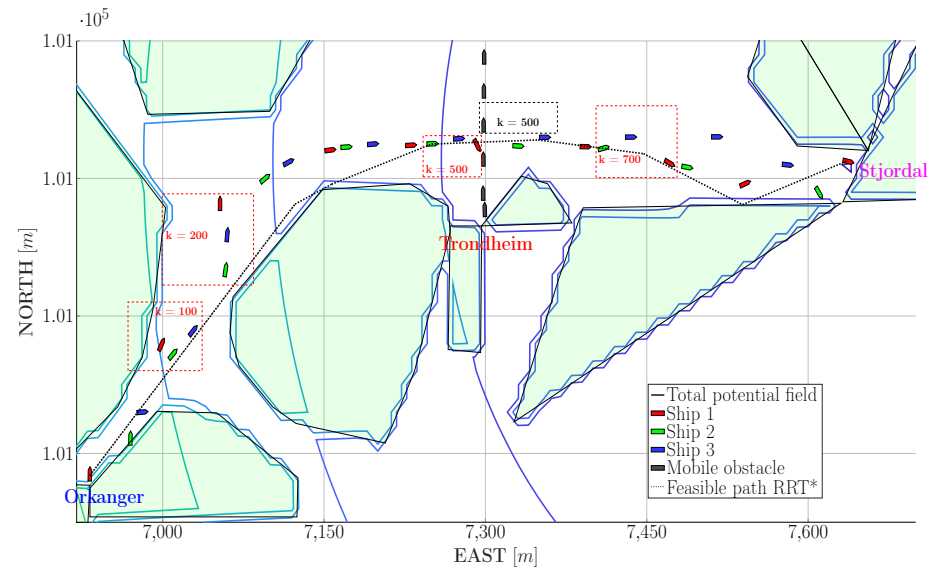

Figure 19: Connectivity maintenance of the three ships group while traveling from Orkanger to Stjordal harbors with COLREGS compliance - rules 8, 13 and 15. 

to Stjordal harbor via RRT* after 1500 iterations as in Fig. 18 .

Fig. 19 illustrates the actual motion of the three vessels traveling from Orkanger to Stjordal harbor. We show the group of agents at 10 different time instances changing their formation configuration due to the moving obstacle illustrated in black.

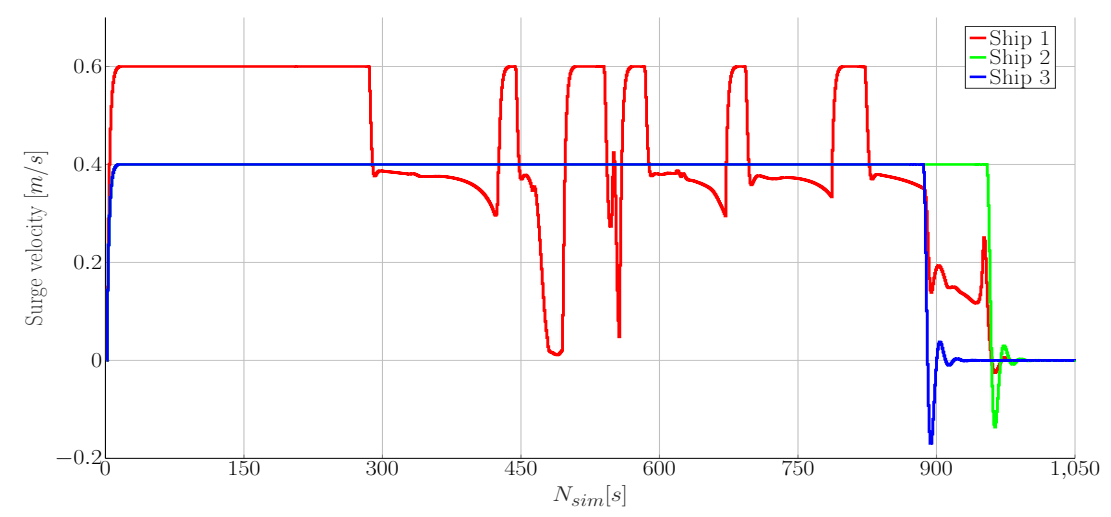

Figure 20: Surge velocities of the three ships in situation 2.
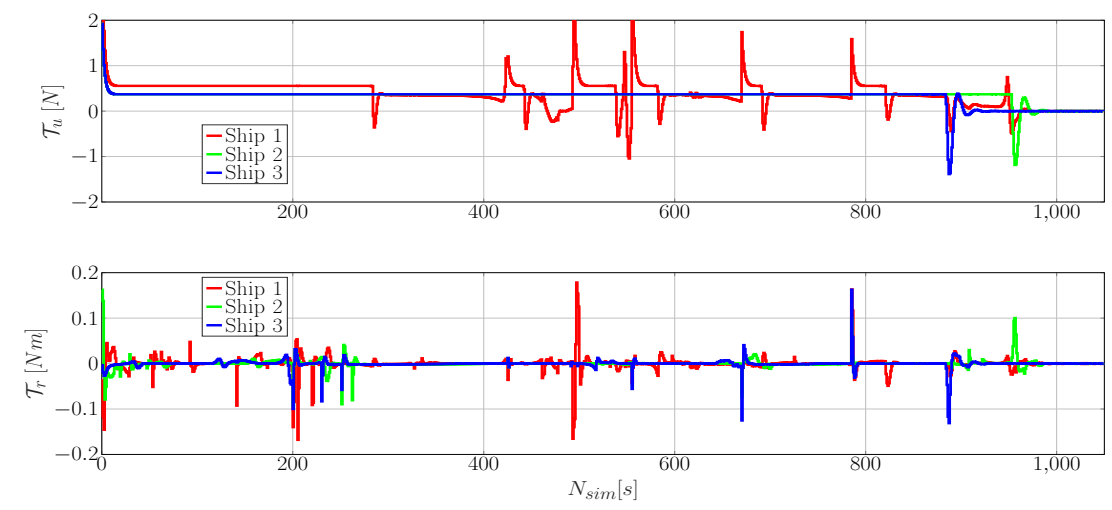

Figure 21: Control inputs of the three ships from Orkanger to Stjordal harbors in situation 2. 


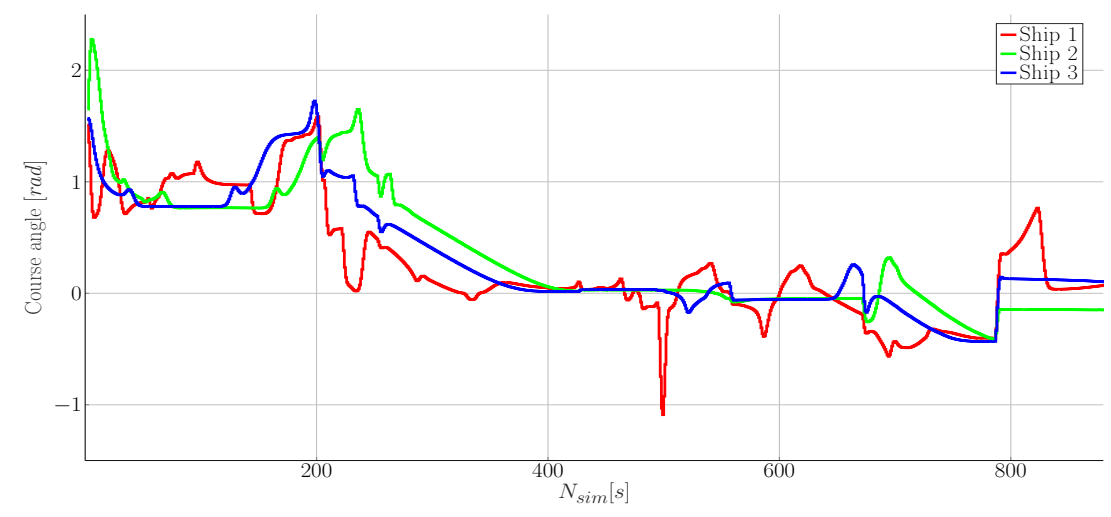

Figure 22: Course angles of the three ships in situation 2.

Note that starting with time step $k=200$, the three ships steer away from the given path due to the repulsive potential of the forbidden cell for ensuring a safe distance between agents and the shore-line. However, after some steps, in particular, at $k=416$, the agents return tracking the given path.

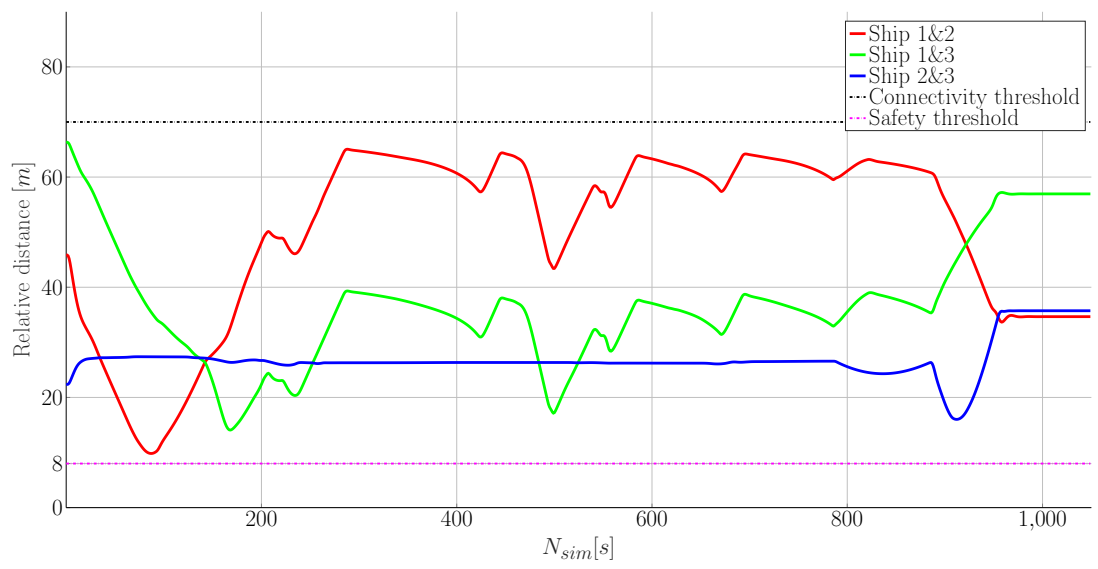

Figure 23: Relative distances between the ships in case of crossing situation with connectivity maintenance.

A crossing situation (Rule 15 of COLREGS) between the group of three ships (which start from Orkanger harbor) and the mobile obstacle (which starts from Trondheim harbor) is encountered. Since the mobile obstacle crosses from 
the starboard of the three ships group, the mobile obstacle can maintain its direction, while the three ships must turn starboard for collision avoidance at time step $k=500$. The change of the ships' course angle can also be observed in Fig. 22, the first (the red line) and third (the blue line) ship's course angles start increasing at time steps $k=500$ and $k=515$, respectively for collision avoidance on starboard with the mobile obstacle. The course angle of the second (the green line) ship is not changing since the mobile obstacle has passed by the time they enter this area.

Fig. 20 and Fig. 21 present the surge velocities and the control input of the three ships respectively.

Collision avoidance and connectivity-preserving among agents are also validated since the relative distances are greater than safety threshold and smaller than the connectivity threshold, as can be seen in Fig. 23

\subsection{Scenario 3}

Assume that the disturbances from the ocean environment are time-varying and are described as follows:

$$
\left\{\begin{array}{l}
w_{u_{i}}=0.96 \sin (0.02 t)+0.84 \sin (0.03 t) \\
w_{v_{i}}=0 \\
w_{r_{i}}=-0.16 \sin \left(0.09 t+\frac{\pi}{3}\right)-0.02 \sin (0.01 t) .
\end{array}\right.
$$

Then the external disturbances are bounded by $\left[\begin{array}{lll}\left\|w_{u_{i}}\right\| & \left\|w_{r_{i}}\right\|\end{array}\right]^{\top} \leq\left[\begin{array}{ll}1.72 & 0.18\end{array}\right]^{\top}$.

\subsubsection{Situation 1}

In this scenario, the NMPC-based optimized control is combined with NDO (eq. (27) and (28) in the presence of the time varying disturbance as in eq. (38). 


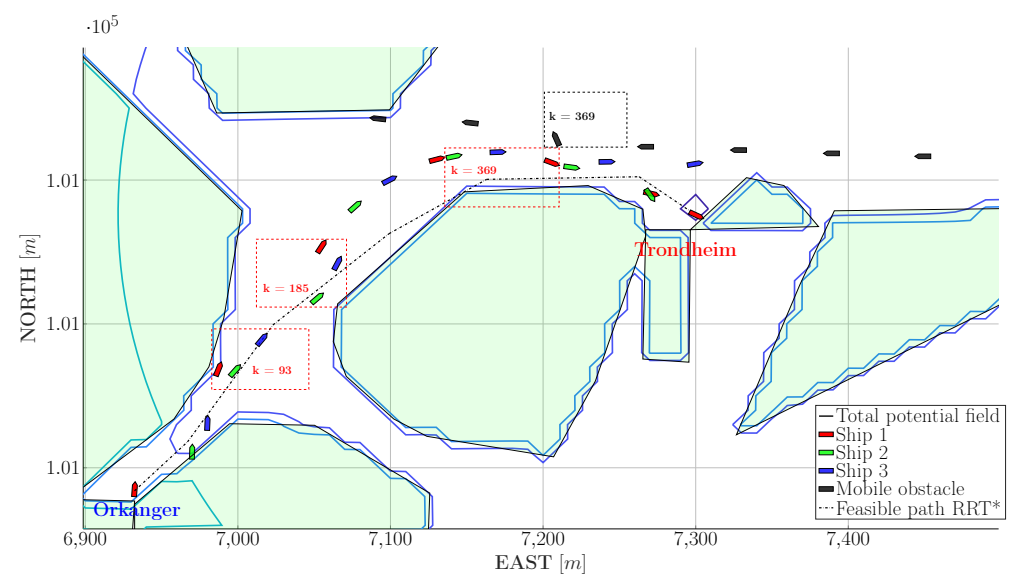

Figure 24: Connectivity maintenance of the three ships group for situation 1 in the presence of disturbances and NDO.

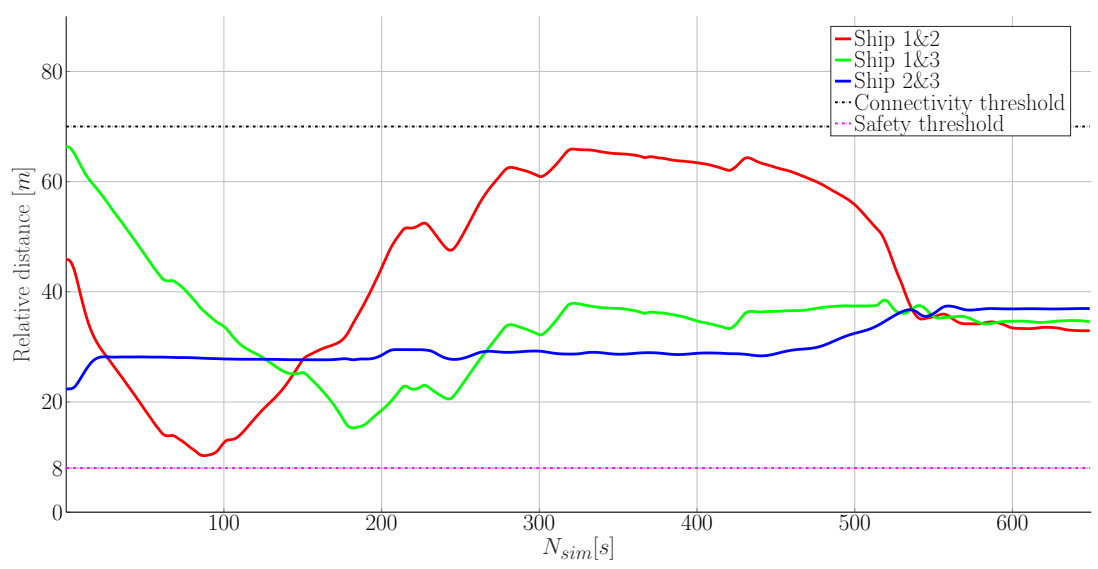

Figure 25: Relative distances of the three agents in situation 1 with the presence of disturbances and NDO.

The effectiveness of the combination between NMPC and NDO is shown in Fig. 24 where the trajectories of three vessels are similar with those shown in situation 1 of scenario 1 . The connectivity maintenance is also guaranteed as observed in Fig. 25 


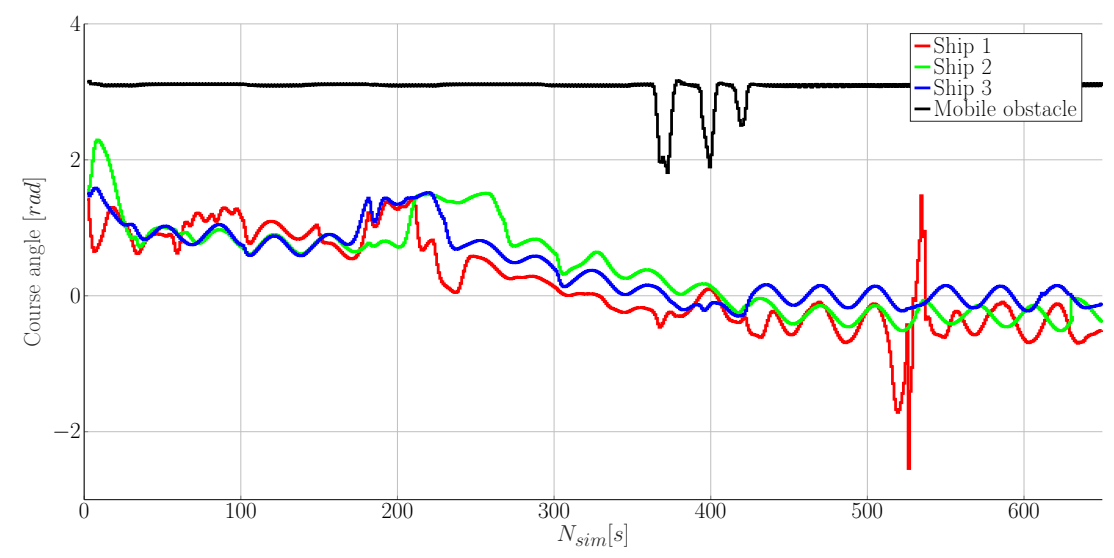

Figure 26: Course angles of the three ships in situation 1 with the presence of disturbances and NDO.

Although the course angle of the three ships is oscillating more than in the case of no disturbance (in Fig. 15), there is also an increased trend for the ships and the mobile obstacle to steer to their starboard side. For example, at the time instance $k=368$, the course angle of ship 1 (red line) and mobile obstacle (black line) increase, which also can be observed in Fig.26 As a consequence, the COLREG rules 13 and 14 are maintained as in situation 1 of Scenario 1.
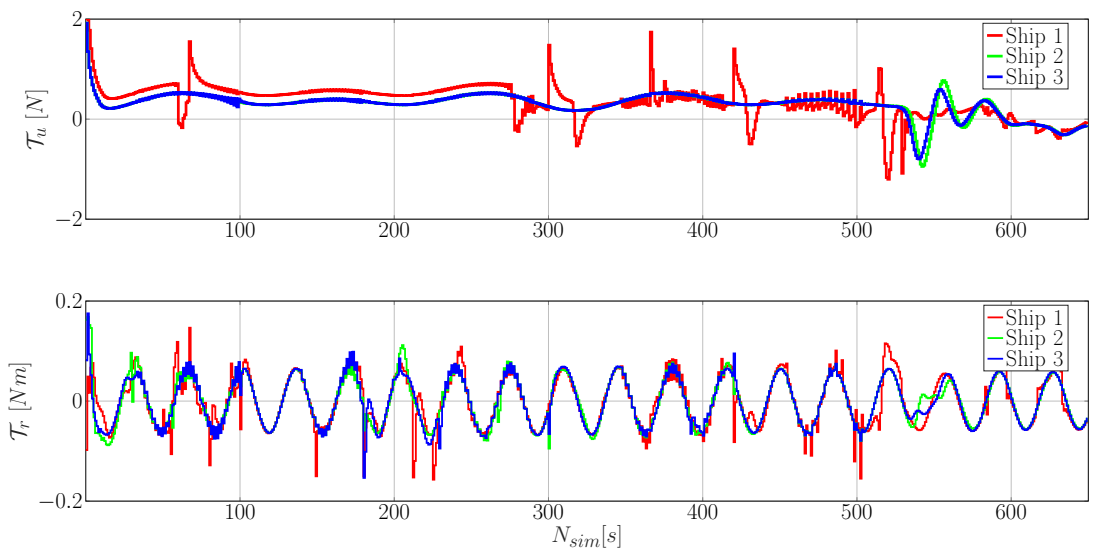

Figure 27: Control inputs of the three ships in situation 1 with the presence of disturbances and NDO. 


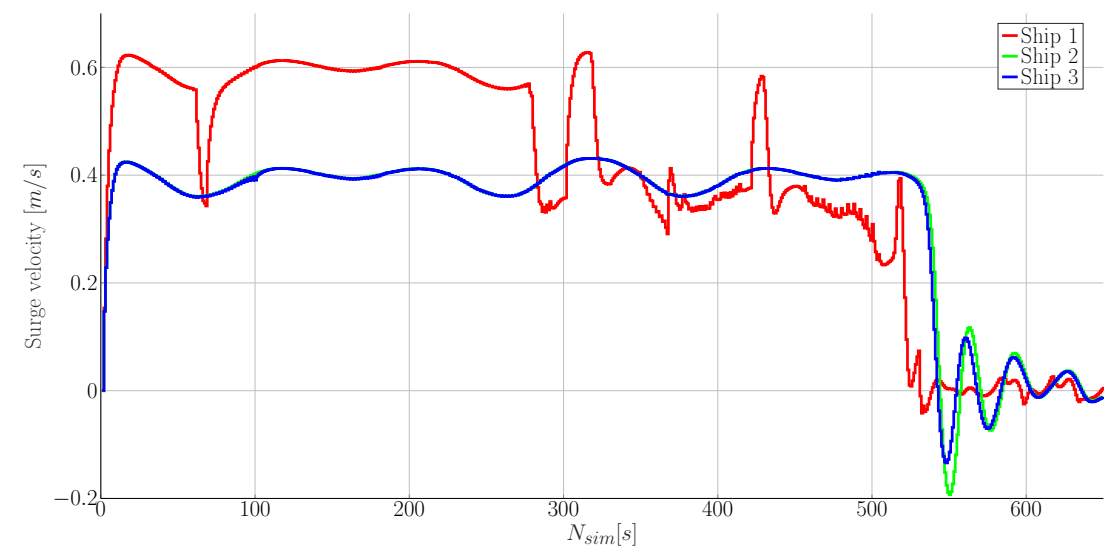

Figure 28: Surge velocities of the three ships in situation 1 with the presence of external disturbances and NDO.

Fig. 27 and 28 the control inputs and surge velocites of the three vessels are presented.
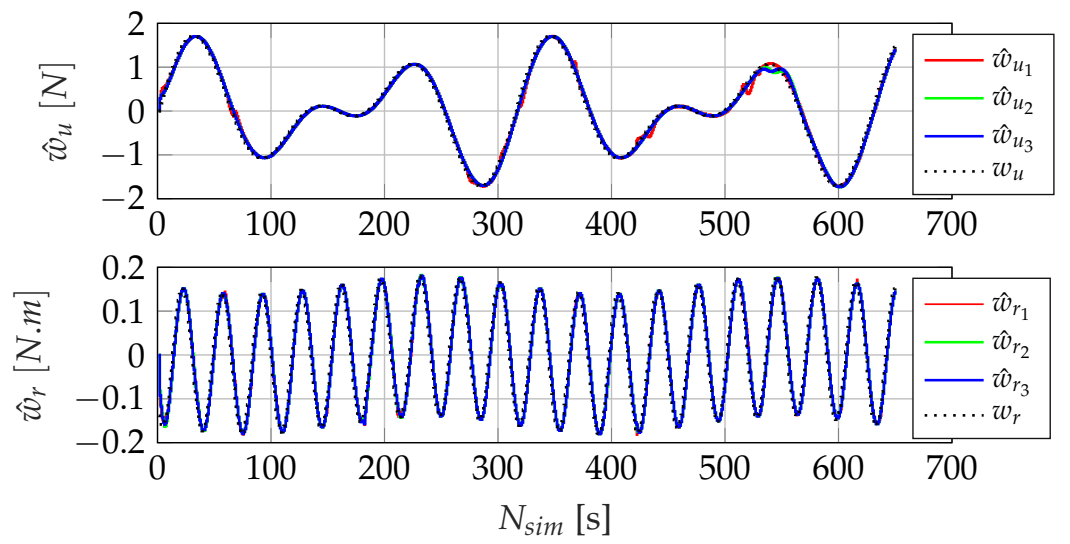

Figure 29: The estimated disturbances of situation 1.

Since the nonlinear disturbances of each ship can be estimated and compensated for, the estimated perturbations $(\hat{w})$ of three ships converge towards 515 the real disorder $(w)$ by depicted in Fig. 29 . The effectiveness of the use of onoff barrier function for the designed function 26) (for example, the NDO of the 
$2^{\text {nd }}$ vessel) as can be seen in Fig. 30. The errors between estimated and actual disturbances when applying the on-off barrier function (the red one) have had shown a slight improvement.
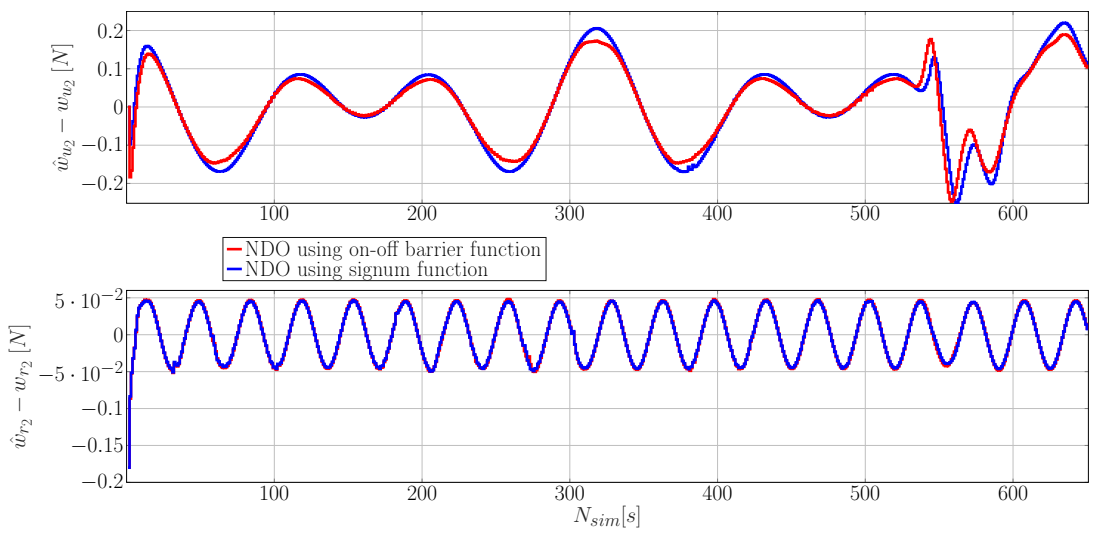

Figure 30: Comparison of NDO using signum function and on-off barrier function.

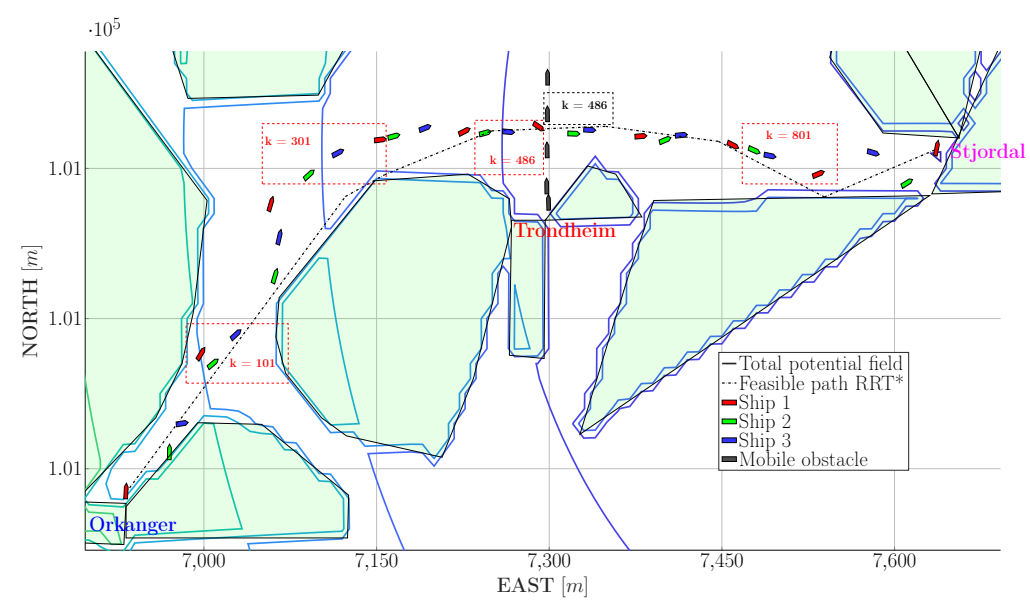

Figure 31: Connectivity maintenance of the three ships group for situation 2 in the presence of external disturbances and NDO. 


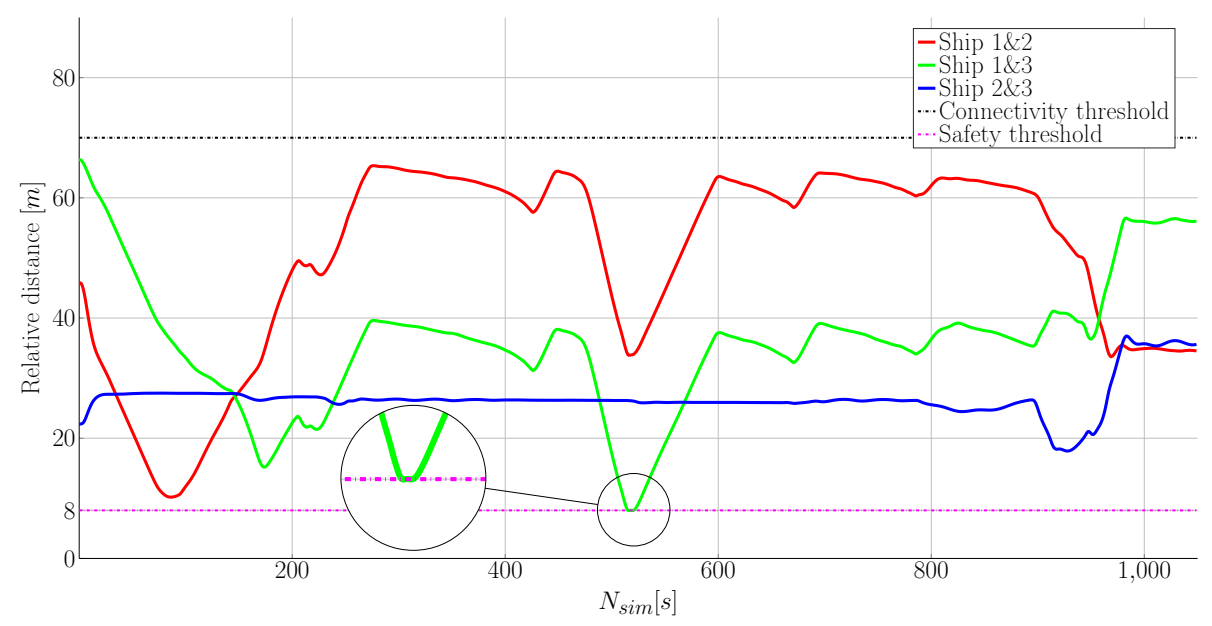

Figure 32: Relative distances of the three agents in situation 2 with the presence of disturbances and NDO.

In this situation, the connectivity maintenance and collision avoidance among agents in the group are also maintained in the simulation as illustrated in Fig. 32 .

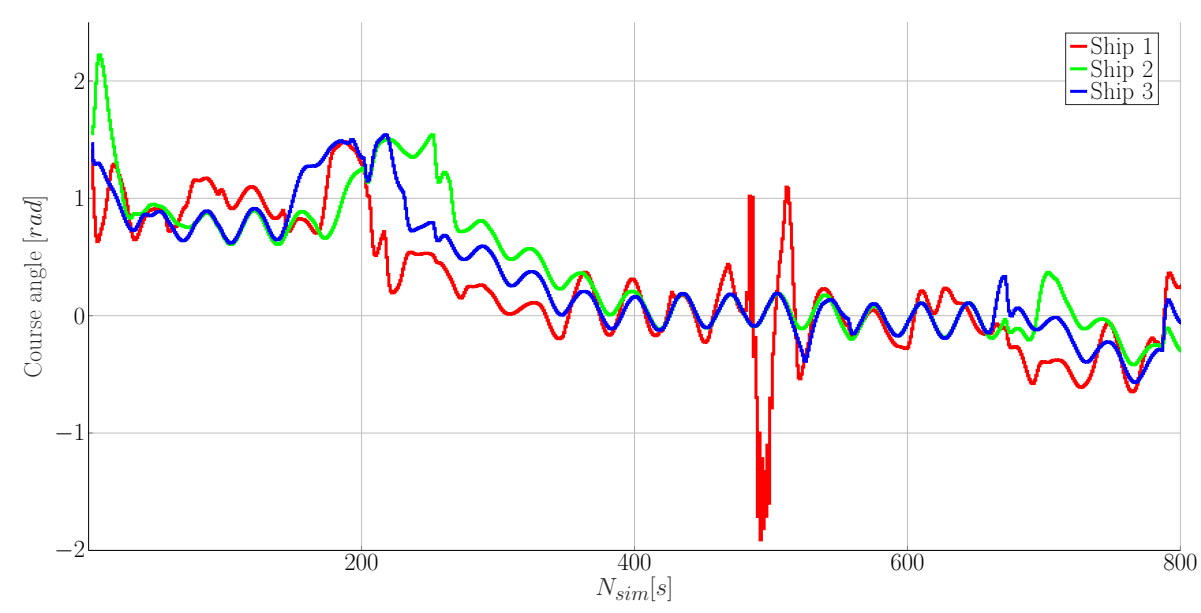

Figure 33: Course angles of the three ships in situation 2 with the presence of disturbances and NDO.

Rule 13 of COLREG - overtaking is obeyed as illustrated in Fig. 31 (at time 

enter this area.

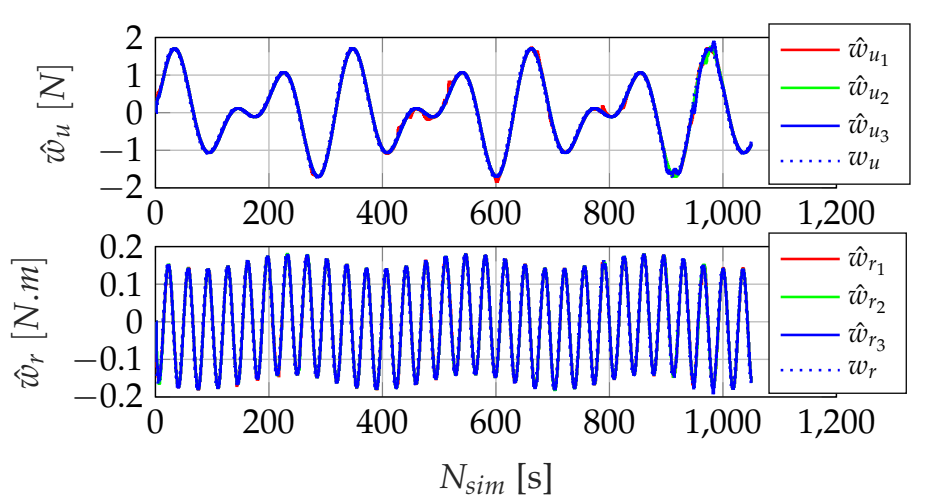

Figure 34: The estimated external disturbances of situation 2.

instance $k=101$, ship 1 in red, overtakes on the left side of the two ships). Moreover, rule 15 - crossing, is also complied when the obstacle ship keeps its direction and ship 1 turns starboard for collision avoidance as can be seen $(k=486)$ in Fig. 31 This can also be seen in Fig. 33, the course angle of ship 1 (red) which increases at $k=486$ while course angles of ship 1 (green) and 3 (blue) are not changing since the mobile obstacle has passed by the time they

Similar to situation 1 in this scenario, good results are obtained because NDO provides an estimate that rapidly converges towards the real disturbances depicted in Fig. 34 . This allows the ships' trajectories in the presence of external disturbances (Fig. 31) to behave like the trajectories in the absence of disturbances (Fig. 19).

Note that in Fig. 13, 19,24 and 31, we also illustrate the repulsive and attractive potentials projected in 2D (the repulsive potentials appear around the forbidden region preventing the ship's collison with the fixed and moving obstacles and the attractive potential is represented by circles around the destination harbor).

Computation time of the optimization problem, trajectory length and number of simulations to arrive at the destination are delineated in Table 2 and 3 . 
Table 2: Performance criteria for the motion planning algorithm of situation 1 for Scenario 2 and 3.

\begin{tabular}{||c|c|c|}
\hline Situation 1 & Scenario 2 & Scenario 3 \\
\hline \hline Prediction horizon [s] & 6 & 6 \\
\hline Number of simulations & 650 & 650 \\
\hline CPU time [s]/step & 0.0039 & 0.0039 \\
\hline Length of trajectory [m] & 505.2 & 503.4 \\
\hline
\end{tabular}

Table 3: Performance criteria for the motion planning algorithm of situation 2 for Scenario 2 and 3.

\begin{tabular}{||c|c|c|}
\hline Situation 2 & Scenario 2 & Scenario 3 \\
\hline \hline Prediction horizon [s] & 6 & 6 \\
\hline Number of simulations & 1050 & 1050 \\
\hline CPU time [s]/step & 0.0103 & 0.0103 \\
\hline Length of trajectory [m] & 847.6 & 858.5 \\
\hline
\end{tabular}

\subsubsection{Situation 3}

In this situation, a group of 4 ships which need to maintain connectivity and avoid collision are considered. The parameters of ship 4 are similar to those of ship 2 and 3 (surge/sway velocity, actuation force, yaw moment). The effectiveness of the proposed method is illustrated in Fig. 35 . At time instance $k=111$, ship 1 (in red) starts overtaking on the port side of the ships 2, 4 (in yellow) and 3 until time instance $k=221$. At time instance $k=380$, ship 1 and the mobile obstacle make a right turn to ensure rule 14 (head-on situation) of COLREGS. Moreover, the communication range and the safe distances among the 4 ships are also guaranteed as can be seen in Fig. 36 . 


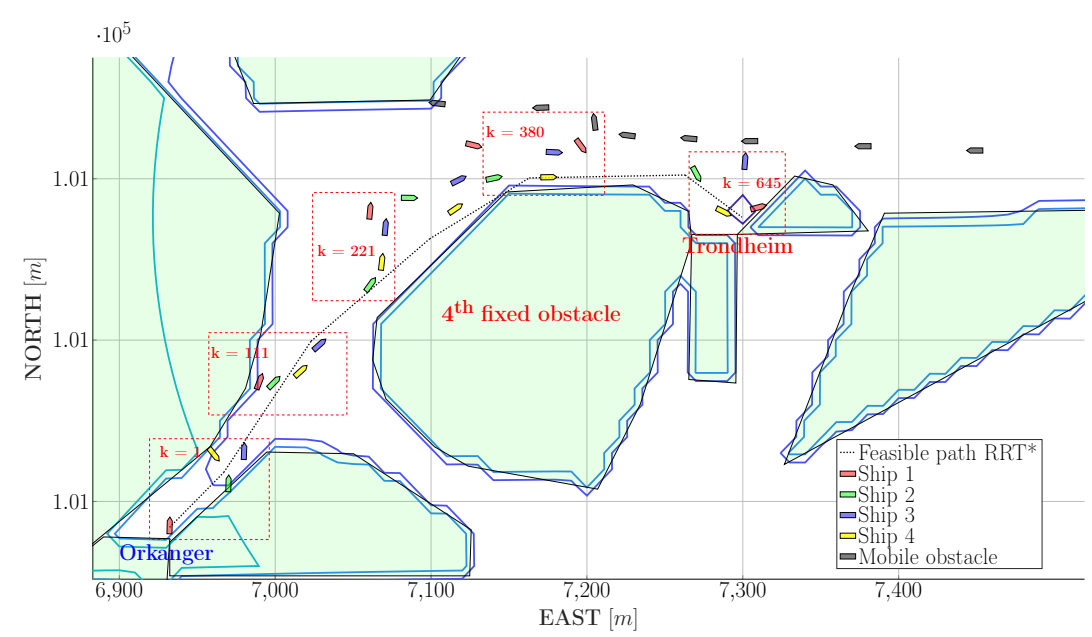

Figure 35: Connectivity maintenance of the four ships group for situation 3 in the presence of disturbances and NDO.

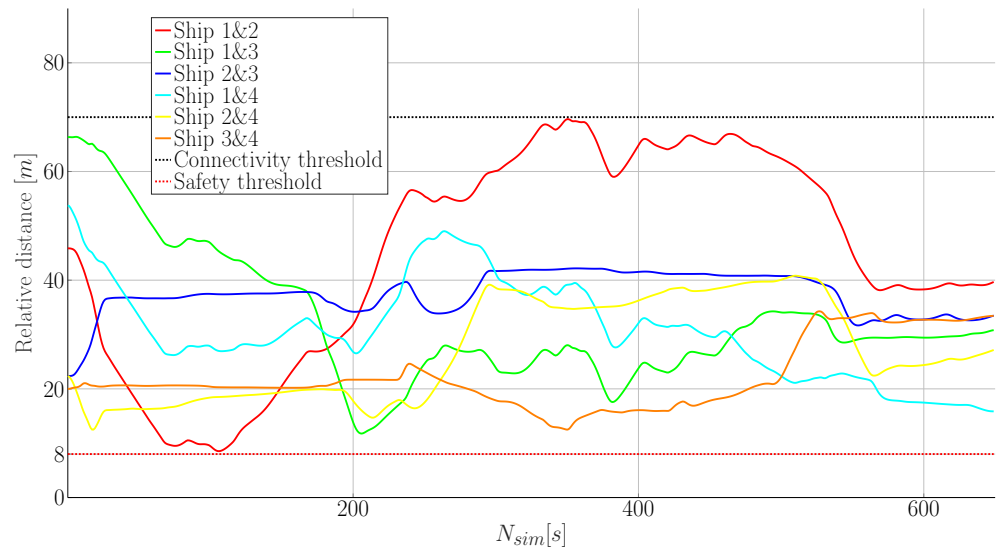

Figure 36: Relative distances of the 4 ships in the group.

It's worth noting that, in this situation, the strength and effect range parameters for the $4^{\text {th }}$ fixed obstacle are descreased. Therefore, the four ships do not meander too far away from the given path when they approach the $4^{\text {th }}$ fixed obstacle with respect to situation 1 of Scenario 2 (Fig. 13) and situation 1 of Scenario 3 (Fig. 24. As we can see at time instance $k=380$, the $4^{\text {th }}$ ship stays on the desired path, while the rest of the ships cannot stay on this path since 


\section{DISCUSSIONS AND CONCLUSIONS}

An NDO-based distributed NMPC scheme has been developed for motion planning problem with connectivity maintenance and collision avoidance for multi-surface vehicles. The classical non-convex constraints from this problem

they must keep a safe distance with ship 4 and with each other. were penalized in the cost function through appropriate potential field constructions. NDO can estimate the external disturbance and compensate for their impact via feedback control.

Although simulation results prove excellent performance of the algorithm as well as give insights for real implementation, there are still some limitations that will be considered in the future work. On one hand, instead of assuming a fixed slope parameter $\beta$, we may obtain it through an estimation/fitting procedure which maximizes the appearance of the obstacles in the agent's view range, i.e., a collision risk may be alerted earlier. The Newton-Raphson [53, 54] and Gradient Descent [55, 56] method as well as its variants such as Batch Gradient Descent and Stochastic Gradient Descent can be employed to estimate this parameter. On the other hand, one of the most challenging perspectives is to guarantee asymptotic stability of the potential field-based MPC scheme by using the predicted input trajectory along the prediction horizon. Without doubt, this is not a trivial problem to deal with since MAS have to leave their predicted paths due to repulsive forces coming from the forbidden areas. Some wellknown approaches from the literature are promising. Stability can be ensured for finite-horizon problems by suitably choosing a weighting matrix for terminal cost and an attractive terminal region [57, 58]. Alternatively, closed-loop stability can also be achieved for relatively long horizons without the need to use a terminal cost or a terminal constraint [59, 60]. Stability is guaranteed by tuning the weighting matrices of the cost function. 


\section{Appendix A}

COLREGS-rules used in the paper:

- Rule 8 - Action to avoid collision: avoidance action must be applied timely, before other vessel approaches. Any alterations of course and/or speed must be large enough to clear the approaching vessels.

- Rule 13 - Overtaking: The overtaking vessel can pass on either side and must keep out of the way of the vessel being overtaken. The vessel being overtaken must hold the course and speed until other vessel is past and well clear.

- Rule 14 - Head on situation: when two power-driven vessels are meeting on nearly reciprocal courses so as to involve risk of collision, then alter course to starboard so that each pass on the port side of each other.

- Rule 15 - Crossing situation: when two power-driven vessels are crossing so as to involve risk of collision, the vessel which has the other on her own starboard side shall keep out of the way.

- Rule 16 - Actions by give-way vessel: take early and substantial action to keep well clear.

\section{Appendix B - Sketch of the proof for Proposition 1.}

Assume that the operating space of the $i^{\text {th }}$ agent is the total potential field that is denoted by $W(\cdot): \mathbb{R}^{n} \rightarrow \mathbb{R}_{\geq 0}$, the potential field which is the summation of repulsive and attractive potential fields and is given as:

$$
W\left(p_{i}\right)=\sum_{\ell=1}^{N_{\text {cell }}} S_{\ell}^{\mathrm{fix}}\left(\gamma_{\ell}\left(p_{i}\right)\right)+\Xi\left(p_{i}\right)
$$

where $\Xi\left(p_{i}\right): \mathbb{R}^{n} \rightarrow \mathbb{R}_{\geq 0}$ is attractive potential and $S_{\ell}^{\mathrm{fix}}\left(\gamma_{\ell}\left(p_{i}\right)\right)$ is the repulsive potential (as presented in (7)) with respect to the $\ell^{\text {th }}$ obstacle, $\ell=1, \ldots, N_{\text {cell }}$. 
Therefore, eq. (39) can be detailed as follows:

$$
W\left(p_{i}\right)=\sum_{\ell=1}^{N_{\text {cell }}} \frac{c_{1 \ell}}{\left(c_{2 \ell}+\gamma_{\ell}\left(p_{i}\right)\right)^{2}}+\left\|p_{i}-p_{i, r e f}\right\|_{\mathbf{Q}_{i}}^{2} .
$$

The simplest control action, $Z_{i}\left(p_{i}\right) \in \mathbb{R}^{n}$, is given by the descent gradient method were the input is equated with the steepest gradient, as presented in [61]:

$$
Z_{i}\left(p_{i}\right)=-\nabla W\left(p_{i}\right)=-\sum_{\ell=1}^{N_{\text {cell }}}\left[\frac{c_{1 \ell}}{\left(c_{2 \ell}+\gamma_{\ell}\left(p_{i}\right)\right)^{3}}\right] \nabla \sum_{\ell=1}^{N_{\text {cell }}} \gamma_{\ell}\left(p_{i}\right)-2 \mathbf{Q}_{i}\left(p_{i}-p_{i, r e f}\right) \nabla p_{i} .
$$

Local minima will happen when there is a poor choice of the parameters of the repulsive and attractive potentials making these potential components cancel each other. This means that (41) is equal to zero, i.e.,

$$
-\sum_{\ell=1}^{N_{\text {cell }}}\left[\frac{c_{1 \ell}}{\left(c_{2 \ell}+\gamma_{\ell}\left(p_{i}\right)\right)^{3}}\right] \nabla \sum_{\ell=1}^{N_{\text {cell }}} \gamma_{\ell}\left(p_{i}\right)=2 \mathbf{Q}_{i}\left(p_{i}-p_{i, r e f}\right) \nabla p_{i} \text {. }
$$

Considering the on-off repulsive potential field ${ }^{10}$ proposed in eq. $(8)$, we can write the total potential as:

$$
W\left(p_{i}\right)=\sum_{\ell=1}^{N_{\text {cell }}} F_{i, \ell}\left(\Delta p_{i, \ell}^{s}, D_{s}^{i, \ell}\right) \frac{c_{1 \ell}}{\left(c_{2 \ell}+\gamma_{\ell}\left(p_{i}\right)\right)^{2}}+\left\|p_{i}-p_{i, r e f}\right\|_{\mathbf{Q}^{\prime}}^{2}
$$

with $F_{i, \ell}(\cdot)$ as in eq. 99.

Next, applying the descent gradient method we calculate the simplest control action given by the steepest gradient of the total potential in eq. (43):

$$
\begin{aligned}
Z_{i}\left(p_{i}\right) & =-\nabla W\left(p_{i}\right)=-\left[-\sum_{\ell=1}^{N_{\text {cell }}} \nabla F_{i, \ell}\left(\Delta p_{i, \ell}^{s}, D_{s}^{i, \ell}\right) \frac{c_{1 \ell}}{\left(c_{2 \ell}+\gamma_{\ell}\left(p_{i}\right)\right)^{2}}\right. \\
& \left.+\sum_{\ell=1}^{N_{\text {obs }}} F_{i, \ell}\left(\Delta p_{i, \ell}^{s}, D_{s}^{i, \ell}\right) \frac{c_{1 \ell}}{\left(c_{2 \ell}+\gamma_{\ell}\left(p_{i}\right)\right)^{3}} \nabla \sum_{\ell=1}^{N_{\text {cell }}} \gamma_{\ell}\left(p_{i}\right)-2 \mathbf{Q}_{i}\left(p_{i}-p_{i, r e f}\right) \nabla p_{i}\right],
\end{aligned}
$$

\footnotetext{
${ }^{10}$ It is worth noting that all the parameters $\left(c_{1 \ell}, c_{2 \ell}\right.$ and $\left.\mathbf{Q}_{i}\right)$ of the total potential field have identical values.
} 
where (44) will become (41) (i.e., local minima) if and only if $\sum_{\ell=1}^{N_{\text {cell }}} F_{i, \ell}(\cdot)=1$ and $\sum_{\ell=1}^{N_{\text {cell }}} \nabla F_{i, \ell}(\cdot)=0$, i.e., the entire total static repulsive potential must be in the interior of the view range of $i^{\text {th }}$ agent (i.e., all static repulsive fields must be activated). The chances of this happening are much reduced since the view range of the agent is significantly smaller than the size of the workspace under consideration.

Therefore, (44) always differs from (41) as long as the agent moves (i.e., the local minima area always moves), in other words, the proposed method can better deal with local minima in comparison to the classical approach.

\section{References}

[1] X. Ge, Q.-L. Han, D. Ding, X.-M. Zhang, B. Ning, A survey on recent advances in distributed sampled-data cooperative control of multi-agent systems, Neurocomputing 275 (2018) 1684-1701.

[2] S. Pang, J. Wang, J. Liu, H. Yi, Three-dimensional leader-follower formation control of multiple autonomous underwater vehicles based on lineof-sight measurements using the backstepping method, Proceedings of the Institution of Mechanical Engineers, Part I: Journal of Systems and Control Engineering 232 (7) (2018) 819-829.

[3] G. Conte, D. Scaradozzi, D. Mannocchi, P. Raspa, L. Panebianco, L. Screpanti, Development and experimental tests of a ros multi-agent structure for autonomous surface vehicles, Journal of Intelligent \& Robotic Systems (2018) 1-14.

[4] U. Commandant, International regulations for prevention of collisions at sea, 1972 (72 colregs), US Department of Transportation, US Coast Guard 16672.

[5] I. Prodan, F. Stoican, S. Olaru, S.-I. Niculescu, Mixed-integer represen- 
tations in control design: Mathematical foundations and applications, Springer, 2015.

[6] S. Cafieri, R. Omheni, Mixed-integer nonlinear programming for aircraft conflict avoidance by sequentially applying velocity and heading angle changes, European Journal of Operational Research 260 (1) (2017) 283290.

[7] M. J. Bays, R. D. Tatum, L. Cofer, J. R. Perkins, Automated scheduling and mission visualization for mine countermeasure operations, in: OCEANS 2015-MTS/IEEE Washington, IEEE, 2015, pp. 1-7.

[8] B. H. Gheneti, Reconfigurable autonomous surface vehicles: perception and trajectory optimization algorithms, Ph.D. thesis, Massachusetts Institute of Technology (2019).

[9] N. Q. H. Tran, I. Prodan, L. Lefèvre, Nonlinear optimization for multiagent motion planning in a multi-obstacle environment, in: 21st IEEE Int. Conf. on System Theory, Control and Computing, 2017, pp. 488-493.

[10] K. Shibata, N. Shibata, K. Nonaka, K. Sekiguchi, Model predictive obstacle avoidance control for vehicles with automatic velocity suppression using artificial potential field, IFAC-PapersOnLine 51 (20) (2018) 313-318.

[11] W. Chao, M. Feng, W. Qing, W. Shuwu, A situation awareness approach for usv based on artificial potential fields, in: Transportation Information and Safety (ICTIS), 2017 4th International Conference on, IEEE, 2017, pp. 232-235.

[12] H. Lyu, Y. Yin, Ship's trajectory planning for collision avoidance at sea based on modified artificial potential field, in: Robotics and Automation Engineering (ICRAE), 2017 2nd International Conference on, IEEE, 2017, pp. 351-357. 
[13] S. Wang, M. Fu, Y. Wang, L. Zhao, A multi-layered potential field method for waterjet propelled unmanned surface vehicle local path planning with minimum energy consumption, Polish Maritime Research.

[14] Y. Kuwata, M. T. Wolf, D. Zarzhitsky, T. L. Huntsberger, Safe maritime navigation with COLREGS using velocity obstacles, in: Intelligent Robots and Systems (IROS), 2011 IEEE/RSJ International Conference on, IEEE, 2011, pp. 4728-4734.

[15] H.-T. L. Chiang, L. Tapia, Colreg-rrt: An RRT-based COLREGS-compliant motion planner for surface vehicle navigation, IEEE Robotics and $\mathrm{Au}-$ tomation Letters 3 (3) (2018) 2024-2031.

[16] T. A. Johansen, T. Perez, A. Cristofaro, Ship collision avoidance and COLREGS compliance using simulation-based control behavior selection with predictive hazard assessment, IEEE transactions on intelligent transportation systems 17 (12) (2016) 3407-3422.

[17] S. Li, J. Liu, R. R. Negenborn, F. Ma, Optimizing the joint collision avoidance operations of multiple ships from an overall perspective, Ocean Engineering 191 (2019) 106511.

[18] A. Mohamed, F. Martin, H. Axel, Nonlinear model predictive control for trajectory tracking and collision avoidance of underactuated vessels with disturbances, Ocean Engineering 160 (12) (2018) 168-180.

[19] M. Abdelaal, M. Fränzle, A. Hahn, NMPC-based trajectory tracking and collison avoidance of underactuated vessels with elliptical ship domain, IFAC-PapersOnLine 49 (23) (2016) 22-27.

[20] L. Chen, H. Hopman, R. R. Negenborn, Distributed model predictive control for vessel train formations of cooperative multi-vessel systems, Transportation Research Part C: Emerging Technologies 92 (2018) 101-118. 
[21] S. Li, J. Liu, R. R. Negenborn, Distributed coordination for collision avoidance of multiple ships considering ship maneuverability, Ocean Engineering 181 (2019) 212-226.

[22] A. Filotheou, A. Nikou, D. V. Dimarogonas, Decentralized control of uncertain multi-agent systems with connectivity maintenance and collision avoidance, in: 2018 European Control Conference (ECC), IEEE, 2018, pp. $8-13$.

[23] H. Fang, Y. Wei, J. Chen, B. Xin, Flocking of second-order multiagent systems with connectivity preservation based on algebraic connectivity estimation, IEEE transactions on cybernetics 47 (4) (2017) 1067-1077.

[24] T. Li, R. Zhao, C. P. Chen, L. Fang, C. Liu, Finite-time formation control of under-actuated ships using nonlinear sliding mode control, IEEE transactions on cybernetics 48 (11) (2018) 3243-3253.

[25] R. Soloperto, J. Köhler, M. A. Müller, F. Allgöwer, Collision avoidance for uncertain nonlinear systems with moving obstacles using robust model predictive control, in: 2019 European Control Conference (ECC), IEEE, 2019, pp. 811-817.

[26] D. Q. Mayne, M. M. Seron, S. Raković, Robust model predictive control of constrained linear systems with bounded disturbances, Automatica 41 (2) (2005) 219-224.

[27] F. Ke, Z. Li, C. Yang, Robust tube-based predictive control for visual servoing of constrained differential-drive mobile robots, IEEE Transactions on Industrial Electronics 65 (4) (2018) 3437-3446.

[28] I. Prodan, S. Olaru, C. Stoica, S.-I. Niculescu, Predictive control for tight group formation of multi-agent systems, IFAC Proceedings Volumes 44 (1) (2011) 138-143.

[29] P. Yu, M. Wu, J. She, K.-Z. Liu, Y. Nakanishi, Robust tracking and disturbance rejection for linear uncertain system with unknown state delay 
and disturbance, IEEE/ASME Transactions on Mechatronics 23 (3) (2018) 1445-1455.

[30] M. Wu, F. Gao, P. Yu, J. She, W. Cao, Improve disturbance-rejection performance for an equivalent-input-disturbance-based control system by incorporating a proportional-integral observer, IEEE Transactions on Industrial Electronics 67 (2) (2019) 1254-1260.

[31] J. Yang, W. X. Zheng, Offset-free nonlinear MPC for mismatched disturbance attenuation with application to a static var compensator, IEEE Transactions on Circuits and Systems II: Express Briefs 61 (1) (2014) 49-53.

[32] S. Yu, Y. Guo, L. Meng, T. Qu, H. Chen, MPC for path following problems of wheeled mobile robots, IFAC-PapersOnLine 51 (20) (2018) 247-252.

[33] C. Liu, W.-H. Chen, J. Andrews, Trajectory tracking of small helicopters using explicit nonlinear MPC and DOBC, IFAC Proceedings Volumes 44 (1) (2011) 1498-1503.

[34] K. D. Do, Practical control of underactuated ships, Ocean Engineering 37 (13) (2010) 1111-1119.

[35] Y. Yang, J. Du, H. Liu, C. Guo, A. Abraham, A trajectory tracking robust controller of surface vessels with disturbance uncertainties, IEEE Transactions on Control Systems Technology 22 (4) (2014) 1511-1518.

[36] N. Wang, Z. Sun, J. Yin, S.-F. Su, S. Sharma, Finite-time observer based guidance and control of underactuated surface vehicles with unknown sideslip angles and disturbances, IEEE Access 6 (2018) 14059-14070.

[37] Z. Liu, C. Geng, J. Zhang, Model predictive controller design with disturbance observer for path following of unmanned surface vessel, in: 2017 IEEE International Conference on Mechatronics and Automation (ICMA), IEEE, 2017, pp. 1827-1832. 
[38] N. Q. H. Tran, I. Prodan, E. I. Grøtli, L. Lefèvre, Potential-field constructions in an MPC framework: application for safe navigation in a variable coastal environment, The 6th IFAC Conference on Nonlinear Model Predictive Control (NMPC'18) 343-348.

[39] T. I. Fossen, Marine control systems: guidance, navigation and control of ships, rigs and underwater vehicles, Marine Cybernetics, 2002.

[40] B.-O. H. Eriksen, M. Breivik, E. F. Wilthil, A. L. Flåten, E. F. Brekke, The branching-course model predictive control algorithm for maritime collision avoidance, Journal of Field Robotics 36 (7) (2019) 1222-1249.

[41] N. Kyurkchiev, S. Markov, Approximation of the cut function by some generic logistic functions and applications, Advances in Applied Sciences 1 (2) (2016) 24-29.

[42] E. F. Camacho, C. Bordons, Model predictive control. advanced textbooks in control and signal processing, Springer-Verlag, London.

[43] S. M. LaValle, Rapidly-exploring random trees: A new tool for path planning.

[44] P. Pharpatara, B. Hérissé, R. Pepy, Y. Bestaoui, Shortest path for aerial vehicles in heterogeneous environment using rrt, in: Robotics and Automation (ICRA), 2015 IEEE International Conference on, IEEE, 2015, pp. 6388-6393.

[45] M. Breivik, T. I. Fossen, Guidance laws for planar motion control, in: Decision and Control, 2008. CDC 2008. 47th IEEE Conference on, IEEE, 2008, pp. 570-577.

[46] W.-H. Chen, D. J. Ballance, P. J. Gawthrop, J. O'Reilly, A nonlinear disturbance observer for robotic manipulators, IEEE Transactions on industrial Electronics 47 (4) (2000) 932-938. 
[47] Q. Zhu, J. Ma, Z. Liu, K. Liu, Containment control of autonomous surface vehicles: A nonlinear disturbance observer-based dynamic surface control design, Advances in Mechanical Engineering 9 (10) (2017) 1687814017727384.

[48] J. Liu, X. Chen, D. Muñoz de la Peña, P. D. Christofides, Sequential and iterative architectures for distributed model predictive control of nonlinear process systems, AIChE Journal 56 (8) (2010) 2137-2149.

[49] M. A. Müller, M. Reble, F. Allgöwer, Cooperative control of dynamically decoupled systems via distributed model predictive control, International Journal of Robust and Nonlinear Control 22 (12) (2012) 1376-1397.

[50] E. Fredriksen, K. Y. Pettersen, Global $\kappa$-exponential way-point maneuvering of ships: Theory and experiments, Automatica 42 (4) (2006) 677-687.

775 [51] J. A. E. Andersson, J. Gillis, G. Horn, J. B. Rawlings, M. Diehl, CasADi - A software framework for nonlinear optimization and optimal control, Mathematical Programming Computation.

[52] M. Breivik, et al., MPC-based mid-level collision avoidance for asvs using nonlinear programming, in: the IEEE Conf. on Control Technol. and Apps., 2017, pp. 766-772.

[53] H. Bakari, T. Adegoke, A. Yahya, Application of newton rhapson method to non-linear models, International Journal of Mathematics and Statistics Studies 4 (4) (2016) 21-31.

[54] K. Al-Daffaie, S. Khan, Logistic regression for circular data, in: AIP Conference Proceedings, Vol. 1842, AIP Publishing, 2017, p. 030022.

[55] M. Kim, Y. Song, S. Wang, Y. Xia, X. Jiang, Secure logistic regression based on homomorphic encryption: Design and evaluation, JMIR medical informatics 6 (2) (2018) e19. 
[56] G. Manogaran, D. Lopez, Health data analytics using scalable logistic regression with stochastic gradient descent, International Journal of Advanced Intelligence Paradigms 10 (1-2) (2018) 118-132.

[57] H. Chen, F. Allgöwer, A quasi-infinite horizon nonlinear model predictive control scheme with guaranteed stability, Automatica 34 (10) (1998) 12051217.

[58] N. T. Nguyen, I. Prodan, L. Lefèvre, On the use of a computed-torque control law for the terminal region of an NMPC scheme, in: 2019 19th American Control Conference (ACC), IEEE, 2019, pp. 1008-1013.

[59] L. Grüne, NMPC without terminal constraints, IFAC Proceedings Volumes 45 (17) (2012) 1-13.

[60] L. Grüne, J. Pannek, Nonlinear model predictive control, in: Nonlinear Model Predictive Control, Springer, 2017, pp. 45-69.

[61] O. Khatib, Real-time obstacle avoidance for manipulators and mobile robots, The international journal of robotics research 5 (1) (1986) 90-98. 